\title{
NOTES ON THE DISTRIBUTION OF ROOTS MODULO A PRIME OF A POLYNOMIAL
}

\author{
YOSHIYUKI KITAOKA
}

\begin{abstract}
Let $f(x)$ be a monic polynomial in $\mathbb{Z}[x]$ with roots $\alpha_{1}, \ldots, \alpha_{n}$. We point out the importance of linear relations among $1, \alpha_{1}, \ldots, \alpha_{n}$ over rationals with respect to the distribution of local roots of $f$ modulo a prime. We formulate it as a conjectural uniform distribution in some sense, which elucidates data in previous papers.
\end{abstract}

\section{Communicated by Shigeki Akiyama}

In this note, a polynomial means always a monic one over the ring $\mathbb{Z}$ of integers and the letter $p$ denotes a prime number, unless specified. Let

$$
f(x)=x^{n}+a_{n-1} x^{n-1}+\cdots+a_{0}
$$

be a polynomial of degree $n$. As in the previous papers, we put

$$
\operatorname{Spl}_{X}(f):=\{p \leq X \mid f(x) \text { is fully splitting modulo } p\}
$$

for a positive number $X$ and $\operatorname{Spl}(f):=\operatorname{Spl}_{\infty}(f)$. In this note, we require the following conditions on the local roots $r_{1}, \ldots, r_{n}(\in \mathbb{Z})$ of $f(x) \equiv 0 \bmod p$ for a prime $p \in \operatorname{Spl}(f)$ :

$$
\begin{aligned}
& f(x) \equiv \prod_{i=1}^{n}\left(x-r_{i}\right) \bmod p, \\
& 0 \leq r_{1} \leq r_{2} \leq \cdots \leq r_{n}<p .
\end{aligned}
$$

We can determine local roots $r_{i}$ uniquely with this global ordering. If $f$ is irreducible and of $\operatorname{deg}(f)>1$, and $p$ is sufficiently large, then (2) is equivalent to $0<r_{1}<\cdots<r_{n}<p$. Here, we consider two types of distribution of local roots $r_{i}$ of $f$.

2010 Mathematics Subject Classification: 11K.

Keywords: distribution, polynomial, roots modulo a prime, 


\section{YOSHIYUKI KITAOKA}

Before stating them, let $\alpha_{1}, \ldots, \alpha_{n}$ be roots of a polynomial $f$ in (0) and suppose a linear relation

$$
\sum_{i=1}^{n} m_{i} \alpha_{i}=m \quad\left(m_{i}, m \in \mathbb{Q}\right) .
$$

Let us give three typical examples of a linear relation (3) among roots:

The first example is

$$
\sum_{i=1}^{n} \alpha_{i}=\operatorname{tr}(f) \quad\left(:=-a_{n-1}\right) .
$$

We call a linear relation (3) trivial if $m_{1}=\cdots=m_{n}$, otherwise non-trivial. A trivial relation is reduced to the above. We know that for an irreducible polynomial $f$, there is only a trivial relation if the degree $n$ is prime or the Galois group $\operatorname{Gal}\left(\mathbb{Q}\left(\alpha_{1}, \ldots, \alpha_{n}\right) / \mathbb{Q}\right)$ is $S_{n}$ or $A_{n}(n \geq 6)$ as a permutation group of $\left\{\alpha_{1}, \ldots, \alpha_{n}\right\}$ (Proposition2).

The second is a reducible polynomial

$$
f(x)=g(x) h(x) \quad \text { with } \quad 1<\operatorname{deg} g<\operatorname{deg} f .
$$

There is a non-trivial relation $\sum \beta_{i}=\operatorname{tr}(g)$ for roots $\beta_{i}$ of $g$, since a set of roots of $g$ is a proper subset of roots of $f$.

The third is a decomposable polynomial, that is

$$
f(x)=g(h(x)) \quad \text { with } \quad 1<\operatorname{deg} h<\operatorname{deg} f .
$$

For a root $\beta$ of $g(x)=0$, a set of solutions $\gamma_{i}$ of $h(x)=\beta$ is a proper subset of roots of $f(x)$, and we have a non-trivial relation $\sum \gamma_{i}=\operatorname{tr}(h-\beta)=\operatorname{tr}(h) \in \mathbb{Z}$. Some other examples are given in [3] and in the text.

If the degree of $f$ is less than 6 , there is no non-trivial linear relation except the above two types, as shown below. In case of degree 6 , other non-trivial linear relations appear.

The first subject is a kind of uniformity: Let $f$ be a polynomial in (0) of degree $n$ and put

$$
\hat{\mathfrak{D}}_{n}:=\left\{\left(x_{1} \ldots, x_{n}\right) \in[0,1)^{n} \mid 0 \leq x_{1} \leq \cdots \leq x_{n}<1, \sum_{i=1}^{n} x_{i} \in \mathbb{Z}\right\}
$$

and for a set $D \subset[0,1)^{n}$ with $D=\overline{D^{\circ}}$

$$
\operatorname{Pr}_{D}(f, X):=\frac{\#\left\{p \in \operatorname{Spl}_{X}(f) \mid\left(r_{1} / p, \ldots, r_{n} / p\right) \in D\right\}}{\# \operatorname{Spl}_{X}(f)},
$$

where local roots $r_{i}$ satisfy properties (11), (2).

We expect, under an assumption that a polynomial $f$ has only a trivial linear relation (3) among roots. 


\section{Expectation 1.}

$$
\operatorname{Pr}_{D}(f):=\lim _{X \rightarrow \infty} \operatorname{Pr}_{D}(f, X)=\frac{\operatorname{vol}\left(D \cap \hat{\mathfrak{D}}_{n}\right)}{\operatorname{vol}\left(\hat{\mathfrak{D}}_{n}\right)} .
$$

The set $\hat{\mathfrak{D}}_{n}$ is parametrized by $x_{1}, \ldots, x_{n-1}$, since $x_{n}$ equals $\left\lceil\sum_{i<n} x_{i}\right\rceil-\sum_{i<n} x_{i}$, and the volume of the projection $\mathfrak{D}_{n}$ of $\hat{\mathfrak{D}}_{n}$ to a hyperplane $\mathbb{R}^{n-1}$ defined by $x_{n}=0$ is $1 / n$ !. Here, $\lceil x\rceil$ denotes an integer satisfying $x \leq\lceil x\rceil<x+1$ for a real number $x$.

In case of $\operatorname{tr}(f)=0$, we may suppose that $D$ is limited to a domain on $\hat{\mathfrak{D}}_{n}$ by virtue of $\left(r_{1} / p, \ldots, r_{n} / p\right) \in \hat{\mathfrak{D}}_{n}$, and it is easy to see that the right-hand side of (5) is $\operatorname{vol}(\operatorname{pr}(\mathrm{D})) /\left(1 / \mathrm{n}\right.$ !), where pr is a projection $\left(x_{1}, \ldots, x_{n}\right) \mapsto$ $\left(x_{1}, \ldots, x_{n-1}\right)$.

In general, a polynomial has non-trivial linear relations among roots, and suppose that a system of linear equations

$$
\sum_{i=1}^{n} m_{j, i} \alpha_{i}=m_{j} \quad(j=1, \ldots, t)
$$

is a basis of all linear relations (3) restricted to $m_{j, i}, m_{j} \in \mathbb{Z}$. If $f$ is irreducible, then we see $\left(\sum_{i} m_{j, i}\right) \operatorname{tr}(f)=n m_{j}$. We fix a numbering of roots $\alpha_{i}$ of $f$. For a prime $p \in \operatorname{Spl}(f)$, there is a permutation $\sigma \in S_{n}$ (dependent on $p$ ) such that local roots $r_{i}$ satisfy induced relations (cf. Proposition 1)

$$
\sum_{i} m_{j, i} r_{\sigma(i)} \equiv m_{j} \bmod p \quad\left(1 \leq{ }^{\forall} j \leq t\right)
$$

which implies $\sum_{i} m_{j, i} \cdot r_{\sigma(i)} / p-m_{j} / p \in \mathbb{Z}$. Let $\boldsymbol{x}=\left(x_{1}, \ldots, x_{n}\right) \in[0,1]^{n}$ be an accumulation point of $\left(r_{1} / p, \ldots, r_{n} / p\right)$ with the same permutation $\sigma$ above; then we see that $\boldsymbol{x}$ is in the closure of

$$
\mathfrak{D}(f, \sigma):=\left\{\begin{array}{l|l}
\left(x_{1} \ldots, x_{n}\right) \in[0,1)^{n} \mid \begin{array}{l}
0 \leq x_{1} \leq \cdots \leq x_{n}<1, \\
\sum_{i} m_{j, i} x_{\sigma(i)} \in \mathbb{Z} \text { for } 1 \leq{ }^{\forall} \leq t
\end{array}
\end{array}\right\} .
$$

If $\boldsymbol{x}$ is not in $\mathfrak{D}(f, \sigma)$, then $x_{n}$ is equal to 1 and we neglect the case since we are concerned with the volume. We note that the set $\mathfrak{D}(f, \sigma)$ depends on a numbering of roots $\alpha_{i}$ and may be the same for distinct permutations.

If $f$ has only a trivial linear relation, then $\mathfrak{D}(f, \sigma)$ is nothing but $\hat{\mathfrak{D}}_{n}$.

Put

$$
\operatorname{Spl}_{X}(f, \sigma):=\left\{p \in \operatorname{Spl}_{X}(f) \mid \sum_{i} m_{j, i} r_{\sigma(i)} \equiv m_{j} \bmod p\left(1 \leq{ }^{\forall} \leq t\right)\right\} .
$$

If $\operatorname{Spl}_{\infty}\left(f, \sigma_{1}\right) \cap \operatorname{Spl}_{\infty}\left(f, \sigma_{2}\right)$ is an infinite set, then $\operatorname{Spl}_{\infty}\left(f, \sigma_{1}\right)$ and $\operatorname{Spl}_{\infty}\left(f, \sigma_{2}\right)$ are equal except a finite set. The following is a generalization of Expectation 1. 
Expectation $\mathbf{1}^{\prime}$.

$$
\begin{aligned}
\operatorname{Pr}_{D}(f, \sigma): & =\lim _{X \rightarrow \infty} \frac{\#\left\{p \in \operatorname{Spl}_{X}(f, \sigma) \mid\left(r_{1} / p, \ldots, r_{n} / p\right) \in D\right\}}{\# \operatorname{Spl}_{X}(f, \sigma)} \\
& =\frac{\operatorname{vol}(D \cap \mathfrak{D}(f, \sigma))}{\operatorname{vol}(\mathfrak{D}(f, \sigma))}
\end{aligned}
$$

for a permutation $\sigma$ with $\operatorname{dim} \mathfrak{D}(f, \sigma)=n-t$, and vol is the volume as a set of $\operatorname{dim}$ being $n-t$. With respect to the density of a set $\operatorname{Spl}(f, \sigma)$ of primes, our observation is

ExpeCtation $1^{\prime \prime}$.

$$
\lim _{X \rightarrow \infty} \frac{\# \operatorname{Spl}_{X}(f, \sigma)}{\# \operatorname{Spl}_{X}(f)}=c^{-1} \cdot \operatorname{vol}(\mathfrak{D}(f, \sigma)),
$$

where the constant $c$ is independent of $\sigma$.

We give a remark on the numbering of roots : Since (6) and (77) are equivalent to $\sum_{i=1}^{n} m_{j, \sigma^{-1}(i)} \alpha_{\sigma^{-1}(i)}=m_{j}$ and $\sum_{i=1}^{n} m_{j, \sigma^{-1}(i)} r_{i} \equiv m_{j} \bmod p$, we may assume that $\sigma$ in Expectation $1^{\prime}, 1^{\prime \prime}$ is the identity for any numbering of roots with replacing " $c$ is independent of $\sigma$ " by " $c$ is independent of the numbering of roots of $f$ " in Expectation 1".

We note that for a sufficiently large prime $p$, we see that $0<r_{1}+a_{n-1} / n, r_{n}+$ $a_{n-1} / n<p$, and then $\left(\left(r_{1}+a_{n-1} / n\right) / p, \ldots,\left(r_{n}+a_{n-1} / n\right) / p\right) \in \mathfrak{D}(f, \sigma)$ if $f$ is irreducible and that a point $\left(r_{1} / p, \ldots, r_{n} / p\right)$ is on $\mathfrak{D}(f, \sigma)$ if and only if $a_{n-1}=0$. Is it possible to reduce the problem to the case of trace being 0 , using $g(x):=n^{n} f\left(\left(x-a_{n-1}\right) / n\right)=x^{n}+0 \cdot x^{n-1}+\cdots$ ? The relation between local roots $R_{i}$ of $g(x)$ and $r_{i}$ of $f(x)$ is $R_{i} \equiv n r_{\sigma(i)}+a_{n-1} \bmod p$ for a permutation $\sigma$ dependent on $p$.

The second subject is as follows.

For given integers $L(>1), R_{i}$ with $0 \leq R_{i}<L$ and a prime $p \in \operatorname{Spl}(f)$, we require a following congruence condition besides (11), (2) on the local roots $r_{1}, \ldots, r_{n}$ of $f(x) \equiv 0 \bmod p$ :

$$
r_{i} \equiv R_{i} \bmod L \quad\left(1 \leq{ }^{\forall} i \leq n\right) .
$$

We put

$$
\operatorname{Pr}_{X}\left(f, L,\left\{R_{i}\right\}\right):=\frac{\#\left\{p \in \operatorname{Spl}_{X}(f) \mid(10),(2),(9)\right\}}{\# \operatorname{Spl}_{X}(f)}
$$

and

$$
\operatorname{Pr}\left(f, L,\left\{R_{i}\right\}\right):=\lim _{X \rightarrow \infty} \operatorname{Pr}_{X}\left(f, L,\left\{R_{i}\right\}\right) .
$$

Although the existence of the limit is not proved in this case either, there is no data to deny it. By putting

$$
R_{f}:=a_{n-1}+\sum_{i=1}^{n} R_{i} \quad \text { and } \quad d:=\left(R_{f}, L\right),
$$


our second expectation is as follows:

For a polynomial $f$ of degree $\geq 2$ with only a trivial relation (3)

\section{ExpeCtation 2.}

$$
\operatorname{Pr}\left(f, L,\left\{R_{i}\right\}\right):=\frac{1}{L^{n-1}} \sum_{K, q} \frac{E_{n}(K)}{\left[\mathbb{Q}\left(\zeta_{L}\right): \mathbb{Q}(f) \cap \mathbb{Q}\left(\zeta_{L / d}\right)\right]},
$$

where $K$ runs over a set of integers satisfying

$$
1 \leq K \leq n-1,(K, L)=d,
$$

and $q \in(\mathbb{Z} / L \mathbb{Z})^{\times}$satisfy the conditions

$$
\left\{\begin{aligned}
& R_{f} \equiv K q \bmod L \quad\left(\Leftrightarrow R_{f} / d \equiv K / d \cdot q \bmod L / d\right), \\
& {[[q]]=[[1]] \text { on } \mathbb{Q}(f) \cap \mathbb{Q}\left(\zeta_{L / d}\right) . }
\end{aligned}\right.
$$

Let us explain notations: $E_{n}(k)$ is the volume of the set $\left\{x \in[0,1)^{n-1} \mid\left\lceil x_{1}+\cdots\right.\right.$ $\left.\left.\cdots+x_{n-1}\right\rceil=k\right\} . E_{n}(k)$ is also defined as $E_{n}(k):=A(n-1, k) /(n-1)$ !, using Eulerian numbers $A(n, k)(1 \leq k \leq n)$ defined recursively by

$$
A(1,1)=1, A(n, k)=(n-k+1) A(n-1, k-1)+k A(n-1, k) .
$$

$\zeta_{L}$ is a primitive $L$ th root of unity, and $\mathbb{Q}(f)$ is a Galois extension of the rational number field $\mathbb{Q}$ generated by all roots of $f$. For an abelian field $F$ in $\mathbb{Q}\left(\zeta_{c}\right)$ and an integer $a$ relatively prime to $c$, $[[a]]$ denotes an automorphism of $F$ induced by $\zeta_{c} \rightarrow \zeta_{c}^{a}$.

Expectations 1, 2 are supported by numerical data by computer for irreducible and indecomposable polynomials of degree $<6([6,[7])$, which are polynomials with only a trivial linear relation among roots. Expectation 2 fails for some polynomials of $\operatorname{deg} f=6$ with non-trivial linear relations.

Let us refer to a relation with a one-dimensional distribution of $r_{i} / p(i=$ $1, \ldots, n)$ : Let $f$ be a polynomial of degree $n$ with only a trivial linear relation among roots. Given number $a \in[0,1]$, put $D_{i, a}:=\left\{\left(x_{1}, \ldots, x_{n}\right) \in[0,1)^{n} \mid x_{i} \leq a\right\}$. Then we see

$$
\begin{aligned}
& \lim _{X \rightarrow \infty} \frac{\sum_{p \in \operatorname{Spl}_{X}(f)} \#\left\{i \mid r_{i} / p \leq a, 1 \leq i \leq n\right\}}{n \cdot \# \operatorname{Spl}_{X}(f)} \\
& =\lim _{X \rightarrow \infty} \frac{\sum_{p \in \operatorname{Spl}_{X}(f)} \#\left\{i \mid\left(r_{1} / p, \ldots, r_{n} / p\right) \in D_{i, a}\right\}}{n \cdot \# \operatorname{Spl}_{X}(f)} \\
& =\sum_{i=1}^{n} \operatorname{Pr}_{D_{i, a}}(f) / n \\
& =\sum_{i=1}^{n} \frac{\operatorname{vol}\left(D_{i, a} \cap \hat{\mathfrak{D}}_{n}\right)}{n \cdot \operatorname{vol}\left(\hat{\mathfrak{D}}_{n}\right)} \quad \text { (by Expectation 1) }
\end{aligned}
$$




\section{YOSHIYUKI KITAOKA}

which is equal to $a$, as far as we check approximately by the Monte Carlo method (definitely for $n=2,3$ ), that is we have the equi-distribution of $r_{i} / p$.

Lastly, let us give a relation between Expectation 1 and a series of observations in the references. Let a polynomial $f$ of degree $n$ have only a trivial linear relation among roots, and put, for an integer $k$

$$
D_{k}:=\left\{\left(x_{1}, \ldots, x_{n}\right) \in[0,1)^{n} \mid\left\lceil x_{1}+\cdots+x_{n-1}\right\rceil=k\right\} .
$$

Then, under Expectation 1, we have

$$
\begin{aligned}
& \lim _{X \rightarrow \infty} \frac{\#\left\{p \in \operatorname{Spl}_{X}(f) \mid\left(r_{1}+\cdots+r_{n}-\operatorname{tr}(f)\right) / p=k\right\}}{\# \operatorname{Spl}_{X}(f)} \\
& =\lim _{X \rightarrow \infty} \frac{\#\left\{p \in \operatorname{Spl}_{X}(f) \mid\left\lceil r_{1} / p+\cdots+r_{n-1} / p\right\rceil=k\right\}}{\# \operatorname{Spl}_{X}(f)} \\
& =\lim _{X \rightarrow \infty} \frac{\#\left\{p \in \operatorname{Spl}_{X}(f) \mid\left(r_{1} / p, \ldots, r_{n} / p\right) \in D_{k}\right\}}{\# \operatorname{Spl}_{X}(f)} \quad\left(=\operatorname{Pr}_{D_{k}}(f)\right) \\
& \left.=\frac{\operatorname{vol}\left(\left\{\left(x_{1}, \ldots, x_{n}\right) \in \hat{\mathfrak{D}}_{n} \mid\left\lceil\sum_{i=1}^{n-1} x_{i}\right\rceil=k\right\}\right)}{\operatorname{vol}\left(\hat{\mathfrak{D}}_{n}\right)} \quad \text { (by Expectation } 1\right) \\
& =\frac{\operatorname{vol}\left(\left\{\left(x_{1}, \ldots, x_{n}\right) \in[0,1)^{n} \mid x_{1} \leq \cdots \leq x_{n}, \sum_{i=1}^{n} x_{i}=k\right\}\right)}{\operatorname{vol}\left(\hat{\mathfrak{D}}_{n}\right)} \\
& =\frac{\operatorname{vol}\left(\left\{\left(x_{1}, \ldots, x_{n}\right) \in[0,1)^{n} \mid \sum_{i=1}^{n} x_{i}=k\right\}\right)}{n ! \cdot \operatorname{vol}\left(\left\{\left(x_{1}, \ldots, x_{n}\right) \in[0,1)^{n} \mid x_{1} \leq \cdots \leq x_{n}, \sum_{i=1}^{n} x_{i} \in \mathbb{Z}\right\}\right)} \\
& =\operatorname{vol}\left(\left\{\left(x_{1}, \ldots, x_{n-1}\right) \in[0,1)^{n-1} \mid\left\lceil\sum_{i=1}^{n-1} x_{i}\right\rceil=k\right\}\right) \quad\left(\text { projected to } \mathbb{R}^{n-1}\right) \\
& =E_{n}(k) \text {, }
\end{aligned}
$$

which elucidates most of numerical observations in previous papers. We note that the last vol is the usual volume on $\mathbb{R}^{n-1}$, but others are the one on hyperplanes defined by

$$
\sum_{i=1}^{n} x_{i} \in \mathbb{Z} \quad \text { in } \quad \mathbb{R}^{n} .
$$

We discuss a linear relation among roots in the first section, and in the second section, we correct insufficient data given in [6] with respect to (12) and add new ones. 
When we refer to an explicit value of a density, it is an approximation by computer, unless specified.

\section{Linear relation among roots}

Let $f(x)$ be a polynomial $f$ in (10) of degree $n$ with roots $\alpha_{i}(i=1, \ldots, n)$ and suppose a linear relation (3). We may suppose that $m=0$ in (3) to discuss the non-triviality of a linear relation, if necessary. Because, if $\operatorname{tr}(f)=-a_{n-1}=0$ holds, then taking traces, we have $n m=\left(\sum_{i} m_{i}\right) \operatorname{tr}(f)=0$, hence $m=0$. Otherwise, we have $\sum M_{i} \alpha_{i}=0$ for $M_{i}:=m_{i}+m / a_{n-1}$. The non-triviality of (3) is unchanging by this operation.

Just to make sure, let us see a relation between global relations of roots $\alpha_{i}$ and local relations of roots $r_{i}^{\prime}$ of $f(x) \equiv 0 \bmod p$.

Proposition 1. Let $f(x)$ be a polynomial of degree $n$ with roots $\alpha_{1}, \ldots, \alpha_{n}$ and suppose that it has no multiple roots, and let $g_{j}\left(x_{1}, \ldots, x_{n}\right)(j=1, \ldots, t)$ be polynomials in $\mathbb{Z}\left[x_{1}, \ldots, x_{n}\right]$.

If there are global relations $g_{j}\left(\alpha_{1}, \ldots, \alpha_{n}\right)=0(j=1, \ldots, t)$, then there are roots $r_{i}^{\prime}$ of $f(x) \equiv 0 \bmod p$ satisfy $g_{j}\left(r_{1}^{\prime}, \ldots, r_{n}^{\prime}\right) \equiv 0 \bmod p(j=1, \ldots, t)$ for $p \in \operatorname{Spl}(f)$.

Conversely, if roots $r_{i}^{\prime}$ of $f(x) \equiv 0 \bmod p$ satisfy $g_{j}\left(r_{1}^{\prime}, \ldots, r_{n}^{\prime}\right) \equiv 0 \bmod p$ $(j=1, \ldots, t)$ for infinitely many primes $p \in \operatorname{Spl}(f)$, then there is a permutation $\sigma$ such that $g_{j}\left(\alpha_{\sigma(1)}, \ldots, \alpha_{\sigma(n)}\right)=0(j=1, \ldots, t)$.

Proof. Put $K=\mathbb{Q}(f)$ and take a prime $p \in \operatorname{Spl}(f) . K$ is a Galois extension and a prime $p$ is fully splitting in $K$.

First, assume $g_{j}\left(\alpha_{1}, \ldots, \alpha_{n}\right)=0(j=1, \ldots, t)$ : For a prime ideal $\mathfrak{p}$ of $K$ over $p$, take a rational integer $r_{i}^{\prime}$ satisfying $\alpha_{i} \equiv r_{i}^{\prime} \bmod \mathfrak{p}$, which implies $g_{j}\left(r_{1}^{\prime}, \ldots, r_{n}^{\prime}\right) \equiv$ $0 \bmod \mathfrak{p}$, hence $g_{j}\left(r_{1}^{\prime}, \ldots, r_{n}^{\prime}\right) \equiv 0 \bmod p$. Let us see that $r_{1}^{\prime}, \ldots, r_{n}^{\prime}$ are roots of $f(x) \equiv 0 \bmod p$. We see $0=f\left(\alpha_{i}\right) \equiv f\left(r_{i}^{\prime}\right) \bmod \mathfrak{p}$, hence $f\left(r_{i}^{\prime}\right) \equiv 0 \bmod p$. If $p$ is sufficiently large, then we see that $\alpha_{i} \not \equiv \alpha_{j} \bmod \mathfrak{p}$ for $i \neq j$, hence $r_{i}^{\prime} \not \equiv r_{j}^{\prime} \bmod p$, that is, $r_{1}^{\prime}, \ldots, r_{n}^{\prime}$ are all distinct roots of $f(x) \equiv 0 \bmod p$.

Conversely, suppose that there are infinitely many primes $p \in \operatorname{Spl}(f)$ such that $g_{j}\left(r_{1}^{\prime}, \ldots, r_{n}^{\prime}\right) \equiv 0 \bmod p(j=1, \ldots, t)$ for $\operatorname{roots} r_{i}^{\prime}$ of $f(x) \equiv 0 \bmod p$. For such a prime, we fix any prime ideal $\mathfrak{p}$ over $p$; then there is a permutation $\sigma_{p}$ of $\{1, \ldots, n\}$ such that $\alpha_{\sigma_{p}(i)} \equiv r_{i}^{\prime} \bmod \mathfrak{p}$ as above. We take a permutation $\sigma$ satisfying $\sigma=\sigma_{p}$ for infinitely many primes $p \in \operatorname{Spl}(f)$. For such infinitely many primes $p$, we see $g_{j}\left(\alpha_{\sigma(1)}, \ldots, \alpha_{\sigma(n)}\right) \equiv g_{j}\left(r_{1}^{\prime}, \ldots, r_{n}^{\prime}\right) \equiv 0 \bmod \mathfrak{p}$, hence a global relation $g_{j}\left(\alpha_{\sigma(1)}, \ldots, \alpha_{\sigma(n)}\right)=0(j=1, \ldots, t)$. 
We apply this to linear equations $g_{j}:=\sum_{i=1}^{n} m_{j, i} x_{i}-m_{j}(j=1, \ldots, t)$ (cf. (6), (77) ). The following is a sufficient condition to a polynomial being without non-trivial linear relation.

Proposition 2. Let $f(x)$ be an irreducible polynomial of degree $n$. If $n$ is a prime number $p$, or the Galois group $\operatorname{Gal}(\mathbb{Q}(f) / \mathbb{Q})$ is $S_{n}$ or $A_{n}(n \geq 6)$ as a permutation group of roots of $f$, then $f$ has only a trivial linear relation among roots.

P r o of. First, suppose that the degree of a polynomial $f$ is a prime $p$, and let $\alpha_{1}, \ldots, \alpha_{p}$ be roots of $f$, and suppose a linear relation (3). Adding a trivial relation $\sum \alpha_{i}=\operatorname{tr}(f)$ to (3) if necessary, we may assume that $\sum m_{i} \neq 0$. The Galois group $\operatorname{Gal}(\mathbb{Q}(f) / \mathbb{Q})$ acts faithfully on the set of all roots and contains an element $\sigma$ of order $p$, hence we may assume that $\left(\sigma\left(\alpha_{1}\right), \ldots, \sigma\left(\alpha_{p}\right)\right)=\left(\alpha_{2}, \ldots, \alpha_{p}, \alpha_{1}\right)$. Then from the assumption (3) follows

$$
\left(\begin{array}{cccc}
m_{1} & m_{2} & \ldots & m_{p} \\
m_{p} & m_{1} & \ldots & m_{p-1} \\
\vdots & & & \\
m_{2} & m_{3} & \ldots & m_{1}
\end{array}\right)\left(\begin{array}{c}
\alpha_{1} \\
\alpha_{2} \\
\vdots \\
\alpha_{p}
\end{array}\right)=\left(\begin{array}{c}
m \\
m \\
\vdots \\
m
\end{array}\right) .
$$

Since $\alpha_{i}$ 's are not rational, the determinant of the coefficient matrix of entries $m_{i}$ vanishes, hence we have

$$
\prod_{i=0}^{p-1}\left(m_{1}+\zeta^{i} m_{2}+\zeta^{2 i} m_{3}+\cdots+\zeta^{(p-1) i} m_{p}\right)=0
$$

for a primitive $p$ th $\operatorname{root} \zeta:=\zeta_{p}$ of unity, using a formula for cyclic determinant. By the assumption $\sum m_{i} \neq 0$, we have

$$
m_{1}+\zeta^{i} m_{2}+\zeta^{2 i} m_{3}+\cdots+\zeta^{(p-1) i} m_{p}=0 \text { for some } \quad i \quad(0<i<p),
$$

which implies $m_{1}=\cdots=m_{p}$, that is, (3) is trivial, since $\zeta^{i}$ is still a primitive $p$ th root of unity.

Next, suppose that the Galois group $\operatorname{Gal}(\mathbb{Q}(f) / \mathbb{Q})$ is the symmetric group $S_{n}$. For any $1 \leq i<j \leq n$, there is an automorphism $\sigma$ which induces a transposition of $\alpha_{i}$ and $\alpha_{j}$. Hence we have

$$
m=\left(\sum_{k \neq i, j} m_{k} \alpha_{k}\right)+m_{i} \alpha_{i}+m_{j} \alpha_{j}=\left(\sum_{k \neq i, j} m_{k} \alpha_{k}\right)+m_{i} \alpha_{j}+m_{j} \alpha_{i},
$$

which implies

$$
m_{i}\left(\alpha_{i}-\alpha_{j}\right)=m_{j}\left(\alpha_{i}-\alpha_{j}\right)
$$

By $\alpha_{i} \neq \alpha_{j}$, we have $m_{i}=m_{j}$, thus (3) is trivial. 


\section{DISTRIBUTION OF ROOTS MODULO A PRIME}

Finally, suppose that $\operatorname{Gal}(\mathbb{Q}(f) / \mathbb{Q})$ is the alternative group $A_{n}$ and that (3) is non-trivial. Let us show that coefficients $m_{1}, \ldots, m_{n}$ are mutually distinct, first. Suppose that $m_{1}=m_{2}$; acting an even permutation $\alpha_{1} \rightarrow \alpha_{2} \rightarrow \alpha_{3} \rightarrow \alpha_{1}$ on (3), we have

$$
\begin{aligned}
m_{1} \alpha_{1}+m_{2} \alpha_{2}+m_{3} \alpha_{3} & =m-\sum_{i>3} m_{i} \alpha_{i}, \\
m_{3} \alpha_{1}+m_{1} \alpha_{2}+m_{2} \alpha_{3} & =m-\sum_{i>3} m_{i} \alpha_{i}
\end{aligned}
$$

which imply $\left(m_{1}-m_{3}\right)\left(\alpha_{1}-\alpha_{3}\right)=0$, hence $m_{2}=m_{1}=m_{3}$. Considering other $\alpha_{i}(i>3)$ instead of $\alpha_{3}$, we get $m_{1}=m_{2}=\cdots=m_{n}$, which contradicts the non-triviality of (3). Thus coefficients $m_{i}$ are mutually distinct.

Next, considering even permutations:

$$
\left\{\alpha_{1} \leftrightarrow \alpha_{2}, \alpha_{3} \leftrightarrow \alpha_{4}\right\}, \quad\left\{\alpha_{1} \leftrightarrow \alpha_{3}, \alpha_{2} \leftrightarrow \alpha_{4}\right\}, \quad\left\{\alpha_{1} \leftrightarrow \alpha_{4}, \alpha_{2} \leftrightarrow \alpha_{3}\right\},
$$

we get

$$
\begin{gathered}
\left\{\begin{array}{l}
m_{1} \alpha_{1}+m_{2} \alpha_{2}+m_{3} \alpha_{3}+m_{4} \alpha_{4}=m-\sum_{i>4} m_{i} \alpha_{i}, \\
m_{2} \alpha_{1}+m_{1} \alpha_{2}+m_{4} \alpha_{3}+m_{3} \alpha_{4}=m-\sum_{i>4} m_{i} \alpha_{i},
\end{array}\right. \\
\left\{\begin{array}{l}
m_{3} \alpha_{1}+m_{4} \alpha_{2}+m_{1} \alpha_{3}+m_{2} \alpha_{4}=m-\sum_{i>4} m_{i} \alpha_{i}, \\
m_{4} \alpha_{1}+m_{3} \alpha_{2}+m_{2} \alpha_{3}+m_{1} \alpha_{4}=m-\sum_{i>4} m_{i} \alpha_{i},
\end{array}\right.
\end{gathered}
$$

which imply

$$
\begin{aligned}
& \left(m_{1}-m_{2}\right)\left(\alpha_{1}-\alpha_{2}\right)+\left(m_{3}-m_{4}\right)\left(\alpha_{3}-\alpha_{4}\right)=0, \\
& \left(m_{3}-m_{4}\right)\left(\alpha_{1}-\alpha_{2}\right)+\left(m_{1}-m_{2}\right)\left(\alpha_{3}-\alpha_{4}\right)=0,
\end{aligned}
$$

hence $\left(\alpha_{1}-\alpha_{2}\right)^{2}=\left(\alpha_{3}-\alpha_{4}\right)^{2}$. Similarly, we have $\left(\alpha_{1}-\alpha_{2}\right)^{2}=\left(\alpha_{3}-\alpha_{5}\right)^{2}$. Therefore we get

$$
\begin{aligned}
0 & =\left(\alpha_{3}-\alpha_{4}\right)^{2}-\left(\alpha_{3}-\alpha_{5}\right)^{2} \\
& =\left(\alpha_{3}-\alpha_{4}+\alpha_{3}-\alpha_{5}\right)\left(\alpha_{3}-\alpha_{4}-\alpha_{3}+\alpha_{5}\right) \\
& =\left(2 \alpha_{3}-\alpha_{4}-\alpha_{5}\right)\left(-\alpha_{4}+\alpha_{5}\right),
\end{aligned}
$$

i.e., $2 \alpha_{3}=\alpha_{4}+\alpha_{5}$, similarly, $2 \alpha_{3}=\alpha_{4}+\alpha_{6}$. Thus we have a contradiction

$$
\alpha_{5}=\alpha_{6}
$$

Proposition 3. Let $f=x^{4}+a_{3} x^{3}+a_{2} x^{2}+a_{1} x+a_{0}$ be an irreducible polynomial. If there is a non-trivial linear relation among roots of $f$, then $f$ is decomposable, that is $f(x)=g(h(x))$ for quadratic polynomials $g(x), h(x)$. 


\section{YOSHIYUKI KITAOKA}

Pr o of. Let $\alpha_{1}, \ldots, \alpha_{4}$ be the roots of $f$. Let $G:=\operatorname{Gal}(\mathbb{Q}(f) / \mathbb{Q})$ be the Galois group; then it operates faithfully on a set $\left\{\alpha_{1}, \ldots, \alpha_{4}\right\}$ and there is a subgroup of order 4 in $G$. Noting that for permutations:

$$
\begin{aligned}
& \sigma=(1,2), \quad \mu=(1,3) \quad \Rightarrow \quad \sigma \mu \neq \mu \sigma, \\
& \sigma=(1,2)(3,4), \quad \mu=(2,3) \quad \Rightarrow \quad \sigma \mu \neq \mu \sigma, \\
& \sigma=(1,2)(3,4), \quad \mu=(1,3)(2,4) \Rightarrow \sigma \mu=\mu \sigma \text {, }
\end{aligned}
$$

we see that:

(i) there is a cyclic permutation $\sigma$ in $G$ so that

$$
\sigma:\left(\alpha_{1}, \alpha_{2}, \alpha_{3}, \alpha_{4}\right) \rightarrow\left(\alpha_{2}, \alpha_{3}, \alpha_{4}, \alpha_{1}\right),
$$

(ii) there are permutations $\sigma_{1}, \sigma_{2}$ in $G$ so that

$$
\begin{aligned}
& \sigma_{1}:\left(\alpha_{1}, \alpha_{2}, \alpha_{3}, \alpha_{4}\right) \rightarrow\left(\alpha_{2}, \alpha_{1}, \alpha_{4}, \alpha_{3}\right), \\
& \sigma_{2}:\left(\alpha_{1}, \alpha_{2}, \alpha_{3}, \alpha_{4}\right) \rightarrow\left(\alpha_{3}, \alpha_{4}, \alpha_{1}, \alpha_{2}\right) \text { or }
\end{aligned}
$$

(iii) there are permutations $\sigma_{1}, \sigma_{2}$ such that $\sigma_{1}$ (resp. $\sigma_{2}$ ) is a transposition of $\alpha_{1}$ and $\alpha_{2}\left(\alpha_{3}\right.$ and $\left.\alpha_{4}\right)$, respectively.

Suppose that (3) is non-trivial, that is ${ }^{\exists} m_{i} \neq{ }^{\exists} m_{j}$, and if $a_{3}=0$ happens, then considering $f(x-1)$ instead of $f(x)$, we may assume that $a_{3} \neq 0$ and furthermore $\sum m_{i} \neq 0$, adding a trivial relation.

First, let us consider

Case (i). By linear equations $\sum m_{i} \sigma^{j}\left(\alpha_{i}\right)=m(j=0,1,2,3)$, we have

$$
\left(\begin{array}{llll}
m_{1} & m_{2} & m_{3} & m_{4} \\
m_{4} & m_{1} & m_{2} & m_{3} \\
m_{3} & m_{4} & m_{1} & m_{2} \\
m_{2} & m_{3} & m_{4} & m_{1}
\end{array}\right)\left(\begin{array}{l}
\alpha_{1} \\
\alpha_{2} \\
\alpha_{3} \\
\alpha_{4}
\end{array}\right)=\left(\begin{array}{llll}
\alpha_{1} & \alpha_{2} & \alpha_{3} & \alpha_{4} \\
\alpha_{2} & \alpha_{3} & \alpha_{4} & \alpha_{1} \\
\alpha_{3} & \alpha_{4} & \alpha_{1} & \alpha_{2} \\
\alpha_{4} & \alpha_{1} & \alpha_{2} & \alpha_{3}
\end{array}\right)\left(\begin{array}{l}
m_{1} \\
m_{2} \\
m_{3} \\
m_{4}
\end{array}\right)=\left(\begin{array}{l}
m \\
m \\
m \\
m
\end{array}\right) .
$$

Since $\alpha_{i}$ 's are irrational, the determinant of coefficient matrix on $m_{i}$ vanishes, i.e., $\prod_{i=0}^{3}\left(m_{1}+\zeta^{i} m_{2}+\zeta^{2 i} m_{3}+\zeta^{3 i} m_{4}\right)=0$ for a primitive fourth root $\zeta:=\zeta_{4}$ of unity. By the assumption $\sum m_{i} \neq 0$, we have

hence

$$
m_{1}+\zeta^{i} m_{2}+\zeta^{2 i} m_{3}+\zeta^{3 i} m_{4}=0 \text { for some } i=1,2,3,
$$

(i.1) $m_{1}-m_{3}=m_{2}-m_{4}=0$ in the case of $i=1,3$ or

(i.2) $m_{1}-m_{2}+m_{3}-m_{4}=0$ in the case of $i=2$.

Case of (i.1), i.e., $m_{1}=m_{3}, m_{2}=m_{4}$ :

The difference of the first row and the second row gives

$$
\left(m_{1}-m_{2}\right)\left(\alpha_{1}-\alpha_{2}+\alpha_{3}-\alpha_{4}\right)=0 .
$$




\section{DISTRIBUTION OF ROOTS MODULO A PRIME}

If $m_{1}=m_{2}$ holds, we have a contradiction $m_{1}=\cdots=m_{4}$. It implies $\alpha_{1}+$ $\alpha_{3}=\alpha_{2}+\alpha_{4}=-a_{3} / 2$, hence $f(x)=\left(x^{2}+a_{3} x / 2+\alpha_{1} \alpha_{3}\right)\left(x^{2}+a_{3} x / 2+\alpha_{2} \alpha_{4}\right)$ is a polynomial in $x^{2}+a_{3} x / 2$, that is $f$ is decomposable.

Case of (i.2), hence $m_{1}+m_{3}=m_{2}+m_{4}$ :

It is easy to see that

$$
\left(\begin{array}{cccc}
\alpha_{1} & \alpha_{2} & \alpha_{3} & \alpha_{4} \\
\alpha_{2} & \alpha_{3} & \alpha_{4} & \alpha_{1} \\
\alpha_{3} & \alpha_{4} & \alpha_{1} & \alpha_{2} \\
\alpha_{4} & \alpha_{1} & \alpha_{2} & \alpha_{3}
\end{array}\right)\left(\begin{array}{l}
m_{1}-m_{2} \\
m_{2}-m_{3} \\
m_{3}-m_{4} \\
m_{4}-m_{1}
\end{array}\right)=\left(\begin{array}{l}
0 \\
0 \\
0 \\
0
\end{array}\right) .
$$

By non-triviality $\left(m_{1}-m_{2}, \ldots, m_{4}-m_{1}\right) \neq(0, \ldots, 0)$, the cyclic determinant of coefficients matrix vanishes, i.e.,

$$
a_{3}\left(\alpha_{1}+\zeta \alpha_{2}-\alpha_{3}-\zeta \alpha_{4}\right)\left(\alpha_{1}-\alpha_{2}+\alpha_{3}-\alpha_{4}\right)\left(\alpha_{1}-\zeta \alpha_{2}-\alpha_{3}+\zeta \alpha_{4}\right)=0 .
$$

(i.2.1) Suppose $\alpha_{1}+\zeta \alpha_{2}-\alpha_{3}-\zeta \alpha_{4}=0$, i.e., $\alpha_{1}-\alpha_{3}=-\zeta\left(\alpha_{2}-\alpha_{4}\right)$. By equations $\sum m_{i} \alpha_{i}=m$ and (by acting $\sigma^{2}$ on it) $m_{1} \alpha_{3}+m_{2} \alpha_{4}+m_{3} \alpha_{1}+m_{4} \alpha_{2}=m$, we have

$$
\left(m_{1}-m_{3}\right)\left(\alpha_{1}-\alpha_{3}\right)+\left(m_{2}-m_{4}\right)\left(\alpha_{2}-\alpha_{4}\right)=0,
$$

hence $\left(\left(m_{1}-m_{3}\right)(-\zeta)+m_{2}-m_{4}\right)\left(\alpha_{2}-\alpha_{4}\right)=0$. Therefore we get $m_{1}=m_{3}$, $m_{2}=m_{4}$ and so a contradiction $m_{1}=m_{2}=m_{3}=m_{4}$ by the assumption $m_{1}+m_{3}=m_{2}+m_{4}$.

(i.2.2) Suppose that $\alpha_{1}-\alpha_{2}+\alpha_{3}-\alpha_{4}=0$; it implies $\alpha_{1}+\alpha_{3}=\alpha_{2}+\alpha_{4}$, which implies that $f$ is decomposable as above.

(i.2.3) The case of $\alpha_{1}-\zeta \alpha_{2}-\alpha_{3}+\zeta \alpha_{4}=0$ is similar to (i.2.1).

Thus we have shown that in the case of (i), $f$ is decomposable.

Case (ii). The second case gives the following equations:

$$
\begin{aligned}
& m_{1} \alpha_{1}+m_{2} \alpha_{2}+m_{3} \alpha_{3}+m_{4} \alpha_{4}=m, \\
& m_{2} \alpha_{1}+m_{1} \alpha_{2}+m_{4} \alpha_{3}+m_{3} \alpha_{4}=m, \\
& m_{3} \alpha_{1}+m_{4} \alpha_{2}+m_{1} \alpha_{3}+m_{2} \alpha_{4}=m, \\
& m_{4} \alpha_{1}+m_{3} \alpha_{2}+m_{2} \alpha_{3}+m_{1} \alpha_{4}=m .
\end{aligned}
$$

(13), (14) (resp. (15), (16)) give

$$
\begin{aligned}
& \left(m_{1}+m_{2}\right)\left(\alpha_{1}+\alpha_{2}\right)+\left(m_{3}+m_{4}\right)\left(\alpha_{3}+\alpha_{4}\right)=2 m, \\
& \left(m_{3}+m_{4}\right)\left(\alpha_{1}+\alpha_{2}\right)+\left(m_{1}+m_{2}\right)\left(\alpha_{3}+\alpha_{4}\right)=2 m,
\end{aligned}
$$

hence if $m_{1}+m_{2} \neq m_{3}+m_{4}$ holds, then $\alpha_{1}+\alpha_{2}=\alpha_{3}+\alpha_{4}$ follows, i.e., $f$ is decomposable. Hence we may suppose that $m_{1}+m_{2}=m_{3}+m_{4}$. 


\section{YOSHIYUKI KITAOKA}

Similarly, using (13), (16) (resp. (14), (15)), we may suppose $m_{1}+m_{4}=m_{2}+$ $m_{3}$, and $m_{1}+m_{3}=m_{2}+m_{4}$, using (13), (15) (resp. (14), (16)). These give a contradiction $m_{1}=m_{2}=m_{3}=m_{4}$.

Finally, let us consider:

Case (iii). Acting $\sigma_{1}, \sigma_{2}$ on $\sum m_{i} \alpha_{i}=m$, we have

$$
\begin{aligned}
& m_{1} \alpha_{1}+m_{2} \alpha_{2}+m_{3} \alpha_{3}+m_{4} \alpha_{4}=m, \\
& m_{1} \alpha_{2}+m_{2} \alpha_{1}+m_{3} \alpha_{3}+m_{4} \alpha_{4}=m, \\
& m_{1} \alpha_{1}+m_{2} \alpha_{2}+m_{3} \alpha_{4}+m_{4} \alpha_{3}=m,
\end{aligned}
$$

which implies

$$
\left(m_{1}-m_{2}\right)\left(\alpha_{1}-\alpha_{2}\right)=\left(m_{3}-m_{4}\right)\left(\alpha_{3}-\alpha_{4}\right)=0 .
$$

Since $\alpha_{i}$ 's are distinct, we have

$$
m_{1}=m_{2}, \quad m_{3}=m_{4} .
$$

By $m_{1} \neq m_{3}$, the equations

$$
\left(\alpha_{1}+\alpha_{2}\right)+\left(\alpha_{3}+\alpha_{4}\right)=-a_{3}, \quad m_{1}\left(\alpha_{1}+\alpha_{2}\right)+m_{3}\left(\alpha_{3}+\alpha_{4}\right)=m
$$

imply

$$
b_{1}:=\alpha_{1}+\alpha_{2} \in \mathbb{Q}, \quad b_{2}:=\alpha_{3}+\alpha_{4} \in \mathbb{Q} .
$$

Therefore

$$
f(x)=\left(x^{2}-\left(\alpha_{1}+\alpha_{2}\right) x+\alpha_{1} \alpha_{2}\right)\left(x^{2}-\left(\alpha_{3}+\alpha_{4}\right) x+\alpha_{3} \alpha_{4}\right)
$$

is equal to

$$
x^{4}+a_{3} x^{3}+\left(\alpha_{3} \alpha_{4}+b_{1} b_{2}+\alpha_{1} \alpha_{2}\right) x^{2}-\left(b_{1} \alpha_{3} \alpha_{4}+b_{2} \alpha_{1} \alpha_{2}\right) x+f(0),
$$

hence we have

$$
\alpha_{1} \alpha_{2}+\alpha_{3} \alpha_{4}=a_{2}-b_{1} b_{2}, \quad b_{2} \alpha_{1} \alpha_{2}+b_{1} \alpha_{3} \alpha_{4}=-a_{1} .
$$

If $b_{1} \neq b_{2}$ holds, then solving them, we have $\alpha_{1} \alpha_{2}, \alpha_{3} \alpha_{4} \in \mathbb{Q}$, which implies that $f$ is reducible. Thus we have $b_{1}=b_{2}$ and then $f$ is a polynomial in $x^{2}-b_{1} x$, that is decomposable.

The following is an easy corollary.

Corollary 1. Let $f$ be a polynomial of degree less than 6 and suppose that $f$ has a non-trivial liner relation among roots. Then $f$ is reducible or decomposable. 
Proposition 4. Let $f=\left(x^{2}+a x\right)^{2}+b\left(x^{2}+a x\right)+c(a, b, c \in \mathbb{Q})$ be an irreducible and decomposable polynomial, and put

$$
x^{2}+b x+c=\left(x-\beta_{1}\right)\left(x-\beta_{2}\right), \quad x^{2}+a x-\beta_{i}=\left(x-\alpha_{i, 1}\right)\left(x-\alpha_{i, 2}\right) .
$$

Then equations $\alpha_{i, 1}+\alpha_{i, 2}=-a(i=1,2)$ are a basis of linear relations (3) among roots of $f$.

P r o o f. Let

$$
m_{1,1} \alpha_{1,1}+m_{1,2} \alpha_{1,2}+m_{2,1} \alpha_{2,1}+m_{2,2} \alpha_{2,2}=m \quad\left(m_{i, j}, m \in \mathbb{Q}\right)
$$

be a linear relation. Using $\alpha_{i, 1}+\alpha_{i, 2}=-a$, we may suppose

$$
m_{1,2} \alpha_{1,2}+m_{2,2} \alpha_{2,2}=m .
$$

We have only to show $m_{2,2}=0$, which implies $m_{1,2}=0$, hence we complete the proof. Suppose that $m_{2,2} \neq 0$, and dividing $m_{2,2}$, we may assume

$$
\alpha_{2,2}=m_{1} \alpha_{1,2}+m_{2} \quad\left(m_{1}, m_{2} \in \mathbb{Q}\right) .
$$

Hence $\alpha_{1,2}$ is a root of $g(x)=x^{2}+a x-\beta_{1}$ and $h(x)=\left(m_{1} x+m_{2}\right)^{2}+$ $a\left(m_{1} x+m_{2}\right)-\beta_{2}=\left(m_{1} x+m_{2}\right)^{2}+a\left(m_{1} x+m_{2}\right)+b+\beta_{1}$, which are polynomials over a quadratic field $\mathbb{Q}\left(\beta_{1}\right)$. Since $g(x)$ is irreducible in $\mathbb{Q}\left(\beta_{1}\right)[x]$, we have $h(x)=m_{1}^{2} g(x)$, hence comparing constant terms $m_{2}^{2}+a m_{2}+b+\beta_{1}=-m_{1}^{2} \beta_{1}$. Thus we find a contradiction that $\beta_{1}$ is rational.

Let us give $\mathfrak{D}(f, \sigma)$ explicitly for a polynomial of degree 4 . In case that $f$ is irreducible and indecomposable, there is only a trivial relation, hence

$$
\mathfrak{D}(f, \sigma)=\hat{\mathfrak{D}}_{n} .
$$

In case that $f$ is irreducible and decomposable, by using Proposition 4, we find, with $\operatorname{dim} \mathfrak{D}(f, \sigma)=2$

$$
\begin{aligned}
& \mathfrak{D}(f, \sigma)=\left\{\begin{array}{l|l}
\left(x_{1}, x_{2}, x_{3}, x_{4}\right) & \begin{array}{l}
0 \leq x_{1} \leq x_{2} \leq x_{3} \leq x_{4} \leq 1, \\
x_{\sigma(1)}+x_{\sigma(2)} \in \mathbb{Z}, x_{\sigma(3)}+x_{\sigma(4)} \in \mathbb{Z}
\end{array}
\end{array}\right\} \\
& =\left\{\left(x_{1}, x_{2}, 1-x_{2}, 1-x_{1}\right) \mid 0 \leq x_{1} \leq x_{2} \leq 1-x_{2} \leq 1-x_{1}<1\right\} \text {, }
\end{aligned}
$$

which is parametrized by $0 \leq x_{1} \leq x_{2} \leq 1 / 2$. The dimension of a domain corresponding to $\{\sigma(1), \sigma(2)\} \neq\{1,4\},\{2,3\}$ is less than 2. To confirm Expectation $1^{\prime}$, i.e., (8), what we can do now is an approximate calculation by computer. The right hand of (8) is a ratio, hence we do not need to look for volumes themselves. By using a projection to $\left(x_{1}, x_{2}\right)$-plane, we can approximate the right hand of (8) by the Monte Carlo method. 


\section{YOSHIYUKI KITAOKA}

In case that $f$ is a product of two irreducible quadratic polynomials with distinct fundamental discriminants, relations are similar to the previous case and hence $\mathfrak{D}(f, \sigma)$ is the same.

In case that two irreducible quadratic factors have the same fundamental discriminant $D$, e.g., $f(x)=\left(x^{2}-D\right)\left((x-1)^{2}-4 D\right)$, put

$$
\alpha_{1}=\sqrt{D}, \quad \alpha_{2}=-\sqrt{D}, \quad \alpha_{3}=1+2 \sqrt{D}, \quad \alpha_{4}=1-2 \sqrt{D} .
$$

A basis of linear relations (3) among roots are

$$
\alpha_{1}+\alpha_{2}=0, \quad 2 \alpha_{1}-\alpha_{3}=-1, \quad 2 \alpha_{1}+\alpha_{4}=1,
$$

hence hyperplanes necessary in $[0,1)^{4}$ in question are

$$
x_{1}+x_{2}, \quad 2 x_{1}-x_{3}, \quad 2 x_{1}+x_{4} \in \mathbb{Z}
$$

and its permutations of indexes.

Thus we see that $\mathfrak{D}(f, \sigma)$ of $\operatorname{dim} 1$ is one of

$$
\begin{aligned}
& \{(x, 2 x, 1-2 x, 1-x) \mid 0 \leq x<1 / 4\} \text { for } \sigma=\left(\begin{array}{cccc}
1 & 2 & 3 & 4 \\
1 & 4 & 2 & 3
\end{array}\right), \\
& \{(x, 1-2 x, 2 x, 1-x) \mid 1 / 4 \leq x<1 / 3\} \text { for } \sigma=\left(\begin{array}{cccc}
1 & 2 & 3 & 4 \\
1 & 4 & 3 & 2
\end{array}\right) \text {, } \\
& \{(1-2 x, x, 1-x, 2 x) \mid 1 / 3 \leq x<1 / 2\} \text { for } \sigma=\left(\begin{array}{cccc}
1 & 2 & 3 & 4 \\
2 & 3 & 4 & 1
\end{array}\right) \text {. }
\end{aligned}
$$

Let us see that Expectation $1^{\prime \prime}$ is true in this case. It is easy to see that lengths are, in order $\sqrt{10} / 4, \sqrt{10} / 12, \sqrt{10} / 6$. For densities of $\operatorname{Spl}(f, \sigma)$, we invoke [1], 9], that is for an irreducible quadratic polynomial, $r_{1} / p, r_{2} / p$ are equi-distributed. Let $r$ be a root of $r^{2} \equiv D \bmod p$ with $0<r<p / 2$. Then other roots of $f(x) \equiv 0 \bmod p$ are $-r, 1 \pm 2 r \bmod p$, and it is easy to see except for finitely many primes local roots $r_{1}, \ldots, r_{4}$ are in order

$$
\left(r_{1}, \ldots, r_{4}\right)=\left\{\begin{array}{lll}
(r, 1+2 r, p+1-2 r, p-r) & \text { if } & r / p \in[0,1 / 4), \\
(r, p+1-2 r, 1+2 r, p-r) & \text { if } & r / p \in[1 / 4,1 / 3), \\
(p+1-2 r, r, p-r, 1+2 r) & \text { if } & r / p \in[1 / 3,1 / 2) .
\end{array}\right.
$$

The uniformity of $r / p$ implies that densities are proportional to

$$
1 / 4,1 / 3-1 / 4=1 / 12,1 / 2-1 / 3=1 / 6 .
$$

Hence the constant $c$ in Expectation $1^{\prime \prime}$ is independent of $\sigma$.

In case that a polynomial $f$ is a product of irreducible quadratic polynomials with the same fundamental discriminant, Expectation $1^{\prime}, 1^{\prime \prime}$ should be reduced to [1, 9]. 
In case that $f(x)=\prod_{i=1}^{a}\left(x-\alpha_{i}\right) \cdot g(x)$, where $\alpha_{i}$ 's are all rational integer roots of $f$ with $\alpha_{1} \leq \ldots \alpha_{t}<0 \leq \alpha_{t+1} \leq \ldots \alpha_{a}$ It is easy to see that

$$
r_{1}=\alpha_{t+1}, \ldots, r_{a-t}=\alpha_{a}, r_{n+1-t}=p+\alpha_{t}, \ldots, r_{n}=p+\alpha_{1}
$$

for any prime $p \in \operatorname{Spl}(f)$ except finitely many primes. Linear relations among roots are reduced to relations of $g$. Therefore the projection of $\mathfrak{D}(f, \sigma)$ to a hyperplane defined by

$$
x_{1}=\cdots=x_{a}=0 \quad \text { with } \quad \sigma(i)=i \quad(1 \leq i \leq a)
$$

is $\mathfrak{D}\left(g, \sigma_{\mid\{a+1, \ldots, n\}}\right)$ modulo a lower dimensional set, hence the problem is reduced to that of a polynomial $g(x)$ as expected.

Before we discuss the case of degree six, let us introduce a notion "type". For an irreducible polynomial $f$ of degree 6 , we define its type number 2,3 temporarily as follows :

Denote a root of $f$ by $\alpha$. The type number of $f$ is 2 if $\mathbb{Q}(\alpha)$ contains a quadratic subfield $M_{2}$ such that the trace of $\alpha$ to $M_{2}$ is rational.

The type number of $f$ is 3 if $\mathbb{Q}(\alpha)$ contains a cubic subfield $M_{3}$ such that the discriminant $D$ of the monic minimal quadratic polynomial $g_{2}(x)$ of $\alpha$ over $M_{3}$ is rational.

We note that the type number is independent of the choice of a root $\alpha$ of $f$, and type numbers of $f(x), f(x+a)(a \in \mathbb{Q})$ are equal.

Proposition 5. Let $f=x^{6}+a_{5} x^{5}+\cdots+a_{0}$ be an irreducible polynomial of degree 6 with roots $\alpha_{1}, \ldots, \alpha_{6}$ and suppose that there is a non-trivial relation (3).

Then we have:

(i) The extension degree $[\mathbb{Q}(f): \mathbb{Q}]$ is not divisible by 5 .

(ii) If $\mathbb{Q}\left(\alpha_{1}\right)$ is an abelian extension, then $f$ is either of type 2 or 3 , or decomposable.

(iii) If $\mathbb{Q}\left(\alpha_{1}\right)$ is an $S_{3}$ Galois extension, then $f$ is either of type 2 or 3 , decomposable or there are a rational number $c$, two distinct roots $\alpha, \alpha^{\prime}$ of $f$, and a cubic subfield $M_{3}$ such that $\operatorname{tr}_{K / M_{3}}(\alpha)+c \cdot \operatorname{tr}_{K / M_{3}}\left(\alpha^{\prime}\right) \in \mathbb{Q}$ and $\alpha, \alpha^{\prime}$ are not conjugate over $M_{3}$.

P r o o f. Let (3) be a non-trivial relation.

(i) Suppose that the extension degree $[\mathbb{Q}(f): \mathbb{Q}]$ is divisible by 5 ; then there is an automorphism $\sigma$ of order 5 in $\operatorname{Gal}(\mathbb{Q}(f) / \mathbb{Q})$, which acts faithfully on a set $\left\{\alpha_{1}, \ldots, \alpha_{6}\right\}$, hence we may assume that

$$
\sigma\left(\alpha_{i}\right)=\alpha_{i+1}(i=1,2,3,4), \quad \sigma\left(\alpha_{5}\right)=\alpha_{1} \quad \text { and } \quad \sigma\left(\alpha_{6}\right)=\alpha_{6} .
$$




\section{YOSHIYUKI KITAOKA}

Adding a trivial relation, we may assume $\sum_{i=1}^{5} m_{i} \neq 0$

$$
\left(\begin{array}{ccccc}
m_{1} & m_{2} & m_{3} & m_{4} & m_{5} \\
m_{5} & m_{1} & m_{2} & m_{3} & m_{4} \\
m_{4} & m_{5} & m_{1} & m_{2} & m_{3} \\
m_{3} & m_{4} & m_{5} & m_{1} & m_{2} \\
m_{2} & m_{3} & m_{4} & m_{5} & m_{1}
\end{array}\right)\left(\begin{array}{c}
\alpha_{1} \\
\alpha_{2} \\
\alpha_{3} \\
\alpha_{4} \\
\alpha_{5}
\end{array}\right)=\left(m-m_{6} \alpha_{6}\right)\left(\begin{array}{l}
1 \\
1 \\
1 \\
1 \\
1
\end{array}\right)
$$

If the determinant of the coefficient matrix does not vanish, then $\alpha_{1}, \ldots, \alpha_{5}$ are in $\mathbb{Q}\left(\alpha_{6}\right)$, hence $\mathbb{Q}\left(\alpha_{6}\right)=\mathbb{Q}\left(\left\{\alpha_{1}, \ldots, \alpha_{6}\right\}\right)=\mathbb{Q}(f)$ is a Galois extension of degree 6 . This contradicts the assumption. Thus the determinant vanishes, hence there is a fifth root $\zeta$ of unity satisfying $\sum_{i=1}^{5} m_{i} \zeta^{i-1}=0$, i.e., $m_{1}=\cdots=m_{5}$. Thus (3) implies $m_{1}\left(\operatorname{tr}(f)-\alpha_{6}\right)+m_{6} \alpha_{6}=m$, which implies $m_{1}=m_{6}$, that is (3) is a trivial relation, contradicting the assumption. This completes the proof of (i).

(ii) Suppose that $\mathbb{Q}\left(\alpha_{1}\right)$ is an abelian extension, hence the Galois group is generated by an automorphism $\sigma$ of order 6 . We may assume that $\sigma\left(\alpha_{i}\right)=\alpha_{i+1}$, where $\alpha_{j}=\alpha_{k}$ if $j \equiv k \bmod$ 6. Otherwise, there is a fixed root $\alpha_{i}$ by $\sigma$. Let $\zeta=(1+\sqrt{-3}) / 2$ be a primitive sixth root of unity, which satisfies $\zeta^{2}-\zeta+1=0$ and $\zeta^{3}=-1$, and consider central idempotents

which satisfies

$$
\chi_{i}=6^{-1} \sum_{j \bmod 6} \zeta^{i j} \sigma^{j},
$$

$$
\sum_{i \bmod 6} \chi_{i}=1, \chi_{i} \chi_{j}=\delta_{i, j} \chi_{j}
$$

The equation (3) is equivalent to $\chi_{i}(m)=\chi_{i}\left(\sum_{k} m_{k} \alpha_{k}\right)$, hence

$$
0=\chi_{i}(m)=\left(\sum_{k \bmod 6} \zeta^{-i k} m_{k}\right)\left(\sum_{l \bmod 6} \zeta^{i l} \alpha_{l}\right) \quad(i \not \equiv 0 \bmod 6),
$$

using $\chi_{i}\left(\alpha_{k}\right)=6^{-1} \zeta^{-k i} \sum_{l \bmod 6} \zeta^{l i} \alpha_{l}$. Thus for

$$
i \not \equiv 0 \bmod 6, \quad \sum_{k \bmod 6} \zeta^{i k} m_{k}=0 \quad \text { or } \sum_{l \bmod 6} \zeta^{i l} \alpha_{l}=0 \text { occurs. }
$$

If $\sum_{l \bmod 6} \zeta^{i l} \alpha_{l}=0$ holds for every $i=1, \ldots, 5$, then we have

$$
\begin{aligned}
0 & =\sum_{i=1}^{5}\left(\sum_{l \bmod 6} \zeta^{i l} \alpha_{l}\right)=5 \alpha_{6}+\sum_{l=1}^{5}\left(\sum_{i=1}^{5} \zeta^{i l}\right) \alpha_{l} \\
& =5 \alpha_{6}-\sum_{l=1}^{5} \alpha_{l}=6 \alpha_{6}-\operatorname{tr}(f)
\end{aligned}
$$


which implies a contradiction $\alpha_{6} \in \mathbb{Q}$. Hence, we have $\sum_{l \bmod 6} \zeta^{i l} \alpha_{l} \neq 0$ for some $i \not \equiv 0 \bmod 6$, i.e.,

$$
\zeta^{-i} \sum_{k=1}^{6} \zeta^{i k} m_{k}=m_{1}+\zeta^{i} m_{2}+\zeta^{2 i} m_{3}+\cdots+\zeta^{5 i} m_{6}=0
$$

By expressing the above as a linear form of $\zeta$ and 1, the equation (17) is

$$
\begin{cases}m_{1}-m_{3}-m_{4}+m_{6}=m_{2}+m_{3}-m_{5}-m_{6}=0 & (i=1,5), \\ m_{1}-m_{2}+m_{4}-m_{5}=m_{2}-m_{3}+m_{5}-m_{6}=0 & (i=2,4), \\ m_{1}+m_{3}+m_{5}=m_{2}+m_{4}+m_{6} & (i=3) .\end{cases}
$$

Suppose that (17) is true for both $i=1$ and $i=2$ : Then we have

$$
m_{1}=m_{3}=m_{5} \quad \text { and } \quad m_{2}=m_{4}=m_{6} .
$$

If (17) is true for $i=3$ moreover, then (3) is a trivial relation, which is a contradiction. Thus (17) is false for $i=3$, hence $\sum_{l \bmod 6} \zeta^{3 l} \alpha_{l}=0$ follows, that is $\alpha_{1}+\alpha_{3}+\alpha_{5}=\alpha_{2}+\alpha_{4}+\alpha_{6}$. Putting $g=\left(x-\alpha_{1}\right)\left(x-\alpha_{3}\right)\left(x-\alpha_{5}\right)$ and $h=\left(x-\alpha_{2}\right)\left(x-\alpha_{4}\right)\left(x-\alpha_{6}\right)$, coefficients of polynomials $g, h$ are in a quadratic subfield $M_{2}$ fixed by $\sigma^{2}$ and their second leading coefficient $\alpha_{1}+\alpha_{3}+\alpha_{5}=$ $\alpha_{2}+\alpha_{4}+\alpha_{6}=\operatorname{tr}(f) / 2$ is rational, hence $f$ is of type 2 .

Suppose that (17) is true for $i=1$, but false for $i=2,4$ : Hence we have $\sum_{l \bmod 6} \zeta^{i l} \alpha_{l}=0$ for $i=2,4$, which implies two equations

$$
\begin{gathered}
\left(\alpha_{1}-\alpha_{2}+\alpha_{4}-\alpha_{5}\right) \sqrt{-3}+\left(-\alpha_{1}-\alpha_{2}+2 \alpha_{3}-\alpha_{4}-\alpha_{5}+2 \alpha_{6}\right)=0, \\
-\left(\alpha_{1}-\alpha_{2}+\alpha_{4}-\alpha_{5}\right) \sqrt{-3}+\left(-\alpha_{1}-\alpha_{2}+2 \alpha_{3}-\alpha_{4}-\alpha_{5}+2 \alpha_{6}\right)=0,
\end{gathered}
$$

hence $\alpha_{1}+\alpha_{4}=\alpha_{2}+\alpha_{5}=\alpha_{3}+\alpha_{6}$. Thus $f$ is a polynomial in $x^{2}+\left(\alpha_{1}+\alpha_{4}\right) x$, that is decomposable.

Finally, we assume that (17) is false for $i=1$ hence for $i=5$; similarly to the above, we have $\alpha_{1}+\alpha_{2}-\alpha_{4}-\alpha_{5}=0$, i.e., $\alpha_{1}-\alpha_{4}=\alpha_{5}-\alpha_{2}$. Hence we see that the discriminant $\left(\alpha_{1}-\alpha_{4}\right)^{2}$ of a polynomial $\left(x-\alpha_{1}\right)\left(x-\alpha_{4}\right)$ fixed by $\sigma^{3}$ is fixed by $\sigma$, hence rational, that is $f$ is of type 3 .

(iii) Suppose that $K=\mathbb{Q}\left(\alpha_{1}\right)$ is an $S_{3}$-extension: Then there are automorphisms $\sigma, \mu$ and a numbering $\beta_{i, j}(i=1,2, j=1,2,3)$ of roots $\alpha_{i}$ of $f$ such that $\sigma^{3}=\eta^{2}=1, \eta \sigma \eta=\sigma^{2}$ and

$$
\begin{gathered}
\sigma\left(\beta_{i, 1}\right)=\beta_{i, 2}, \sigma\left(\beta_{i, 2}\right)=\beta_{i, 3}, \sigma\left(\beta_{i, 3}\right)=\beta_{i, 1} \quad(i=1,2) \\
\eta\left(\beta_{1,1}\right)=\beta_{2,1}, \eta\left(\beta_{1,2}\right)=\beta_{2,3}, \eta\left(\beta_{1,3}\right)=\beta_{2,2},
\end{gathered}
$$

noting that $\sigma, \eta$ have no fixed root. We divide a proof to several parts. 


\section{YOSHIYUKI KITAOKA}

\section{LEMMA 1.}

(1) If $\sum_{j} \beta_{1, j}$ is rational, then $f$ is of type 2 .

(2) If one of $\left(\beta_{1,1}-\beta_{2,1}\right)^{2},\left(\beta_{1,2}-\beta_{2,3}\right)^{2},\left(\beta_{1,3}-\beta_{2,2}\right)^{2}$ is fixed by $\sigma$, then $f$ is of type 3 .

Proof.

Suppose that $\sum_{i} \beta_{1, j} \in \mathbb{Q}$, and decompose $f$ as $f=g h$ with $g=\prod\left(x-\beta_{1, j}\right)$, $h=\prod\left(x-\beta_{2, j}\right) \in M_{2}[x]$, where $M_{2}$ is a quadratic subfield fixed by $\sigma$. Therefore $f$ is of type 2 . Since polynomials $\left(x-\beta_{1,1}\right)\left(x-\beta_{2,1}\right),\left(x-\beta_{1,2}\right)\left(x-\beta_{2,3}\right)$ and $\left(x-\beta_{1,3}\right)\left(x-\beta_{2,2}\right)$ are fixed by $\eta$, their discriminants are also fixed by $\eta$. Therefore, if the discriminant is fixed by $\sigma$, it is a rational number, that is, $f$ is of type 3 .

LEMMA 2. If there is a non-trivial relation

$$
\sum_{j} m_{1, j} \beta_{1, j}+\sum_{j} m_{2, j} \beta_{2, j}=m \quad\left(m_{i, j}, m \in \mathbb{Q}\right),
$$

then $f$ is of type 2 , or there are rational numbers $m_{1}, m_{2}, m_{3}$ with $m_{i} \neq m_{j}$ for some $i, j$ and $m_{1}+m_{2}+m_{3}=0$ such that

$$
m_{1}\left(\beta_{1,1}+\beta_{2,1}\right)+m_{2}\left(\beta_{1,2}+\beta_{2,3}\right)+m_{3}\left(\beta_{1,3}+\beta_{2,2}\right)=0 .
$$

P r o of. We may suppose that $m=0$ in (18) by the remark at the beginning of this section, and acting $i d, \sigma, \sigma^{2}, \eta, \eta \sigma, \eta \sigma^{2}$ in order, we have

$$
\begin{aligned}
& m_{1,1} \beta_{1,1}+m_{1,2} \beta_{1,2}+m_{1,3} \beta_{1,3}+m_{2,1} \beta_{2,1}+m_{2,2} \beta_{2,2}+m_{2,3} \beta_{2,3}=0, \\
& m_{1,3} \beta_{1,1}+m_{1,1} \beta_{1,2}+m_{1,2} \beta_{1,3}+m_{2,3} \beta_{2,1}+m_{2,1} \beta_{2,2}+m_{2,2} \beta_{2,3}=0, \\
& m_{1,2} \beta_{1,1}+m_{1,3} \beta_{1,2}+m_{1,1} \beta_{1,3}+m_{2,2} \beta_{2,1}+m_{2,3} \beta_{2,2}+m_{2,1} \beta_{2,3}=0, \\
& m_{2,1} \beta_{1,1}+m_{2,3} \beta_{1,2}+m_{2,2} \beta_{1,3}+m_{1,1} \beta_{2,1}+m_{1,3} \beta_{2,2}+m_{1,2} \beta_{2,3}=0, \\
& m_{2,3} \beta_{1,1}+m_{2,2} \beta_{1,2}+m_{2,1} \beta_{1,3}+m_{1,3} \beta_{2,1}+m_{1,2} \beta_{2,2}+m_{1,1} \beta_{2,3}=0, \\
& m_{2,2} \beta_{1,1}+m_{2,1} \beta_{1,2}+m_{2,3} \beta_{1,3}+m_{1,2} \beta_{2,1}+m_{1,1} \beta_{2,2}+m_{1,3} \beta_{2,3}=0 .
\end{aligned}
$$

Equations (20) $+(23),(21)+(24),(22)+(25)$ are

$$
\left(\begin{array}{lll}
m_{1,1}+m_{2,1} & m_{1,2}+m_{2,3} & m_{1,3}+m_{2,2} \\
m_{1,3}+m_{2,3} & m_{1,1}+m_{2,2} & m_{1,2}+m_{2,1} \\
m_{1,2}+m_{2,2} & m_{1,3}+m_{2,1} & m_{1,1}+m_{2,3}
\end{array}\right)\left(\begin{array}{c}
\beta_{1,1}+\beta_{2,1} \\
\beta_{1,2}+\beta_{2,3} \\
\beta_{1,3}+\beta_{2,2}
\end{array}\right)=\left(\begin{array}{l}
0 \\
0 \\
0
\end{array}\right) .
$$




\section{DISTRIBUTION OF ROOTS MODULO A PRIME}

If entries in each row of the coefficient matrix in (26) are the same, that is

$$
\left\{\begin{array}{l}
m_{1,1}+m_{2,1}=m_{1,2}+m_{2,3}=m_{1,3}+m_{2,2}:=a(\text { say }), \\
m_{1,3}+m_{2,3}=m_{1,1}+m_{2,2}=m_{1,2}+m_{2,1}:=b \\
m_{1,2}+m_{2,2}=m_{1,3}+m_{2,1}=m_{1,1}+m_{2,3}:=c
\end{array}\right.
$$

then we have

$$
\left\{\begin{array}{l}
m_{2,3}=a-m_{1,2}=b-m_{1,3}=c-m_{1,1} \\
m_{2,2}=a-m_{1,3}=b-m_{1,1}=c-m_{1,2} \\
m_{2,1}=a-m_{1,1}=b-m_{1,2}=c-m_{1,3}
\end{array}\right.
$$

hence

$$
m_{1,3}=m_{1,1}+b-c=m_{1,1}+a-b=m_{1,1}-a+c,
$$

which imply

$$
b-c=a-b=-a+c, \text { hence } a=b=c .
$$

Therefore, we get $m_{1,1}=m_{1,2}=m_{1,3}$ and $m_{2,1}=m_{2,2}=m_{2,3}$. The nontriviality of (20) implies $m_{1,1} \neq m_{2,1}$, hence comparing it with trivial relation $\sum_{i} \beta_{1, i}+\sum_{i} \beta_{2, i}=\operatorname{tr}(f)$, we see $\sum_{i} \beta_{1, i} \in \mathbb{Q}$. By lemma $1 . f$ is of type 2 .

If there are distinct entries in some row of the coefficient matrix in (26), then putting entries of the row by $m_{1}, m_{2}, m_{3}$, we have

$$
m_{1}\left(\beta_{1,1}+\beta_{2,1}\right)+m_{2}\left(\beta_{1,2}+\beta_{2,3}\right)+m_{3}\left(\beta_{1,3}+\beta_{2,2}\right)=0 .
$$

If $\operatorname{tr}(f) \neq 0$, then taking the trace, we have $\left(m_{1}+m_{2}+m_{3}\right) \operatorname{tr}(f)=0$, which implies $m_{1}+m_{2}+m_{3}=0$. If $\operatorname{tr}(f)=0$, then we have only to replace $m_{i}$ by $m_{i}-\left(m_{1}+m_{2}+m_{3}\right) / 3$.

Thus we have, by (19)

$$
m_{1}\left(\beta_{1,1}+\beta_{2,1}-\beta_{1,3}-\beta_{2,2}\right)+m_{2}\left(\beta_{1,2}+\beta_{2,3}-\beta_{1,3}-\beta_{2,2}\right)=0,
$$

where $m_{1}=m_{2}=0$ does not hold.

We divide the proof to several cases:

(I) Case of $m_{1}=0$ and $m_{2} \neq 0$ : We have $\beta_{1,2}+\beta_{2,3}-\beta_{1,3}-\beta_{2,2}=0$, and $\beta_{1,3}+\beta_{2,1}-\beta_{1,1}-\beta_{2,3}=0$, acting $\sigma$. Therefore we find that $\beta_{1,1}-\beta_{2,1}=$ $\beta_{1,3}-\beta_{2,3}=\beta_{1,2}-\beta_{2,2}$, which is $\sigma$-invariant, hence by Lemma 1, $f$ is of type 3 .

(II) Case of $m_{1} \neq 0$ and $m_{2}=0$ : We have $\sigma\left(\beta_{1,1}+\beta_{2,1}-\beta_{1,3}-\beta_{2,2}\right)=\beta_{1,2}+$ $\beta_{2,2}-\beta_{1,1}-\beta_{2,3}=0$, and $\beta_{2,3}+\beta_{1,3}-\beta_{2,1}-\beta_{1,2}=0$, acting $\eta$. Hence, we find that $\sigma\left(\beta_{1,2}-\beta_{2,3}\right)^{2}-\left(\beta_{1,2}-\beta_{2,3}\right)^{2}=\left(\beta_{1,3}-\beta_{2,1}+\beta_{1,2}-\beta_{2,3}\right)\left(\beta_{1,3}-\beta_{2,1}-\beta_{1,2}+\beta_{2,3}\right)=0$ and so by Lemma 1, $f$ is of type 3 .

(III) Case of $m_{1} m_{2} \neq 0$ : Dividing by $m_{2}$, we may suppose $m_{2}=1$, that is

$$
m_{1}\left(\beta_{1,1}+\beta_{2,1}-\beta_{1,3}-\beta_{2,2}\right)+\left(\beta_{1,2}+\beta_{2,3}-\beta_{1,3}-\beta_{2,2}\right)=0
$$




\section{YOSHIYUKI KITAOKA}

Suppose that $m_{1}=1$ : Adding $3\left(\beta_{1,3}+\beta_{2,2}\right)$ to the above, we have $\operatorname{tr}(f)=$ $3\left(\beta_{1,3}+\beta_{2,2}\right)$. Acting $\sigma$, we have $\beta_{1,3}+\beta_{2,2}=\beta_{1,1}+\beta_{2,3}=\beta_{1,2}+\beta_{2,1}=\operatorname{tr}(f) / 3$. Therefore, $f=\left(x-\beta_{1,3}\right)\left(x-\beta_{2,2}\right) \cdot\left(x-\beta_{1,1}\right)\left(x-\beta_{2,3}\right) \cdot\left(x-\beta_{1,2}\right)\left(x-\beta_{2,1}\right)$ is a polynomial in $x^{2}-\operatorname{tr}(f) / 3 \cdot x$, that is decomposable.

Finally, we assume that $m_{1} \neq 1$. Substituting $\beta_{1,2}+\beta_{2,3}=-\left(\beta_{1,1}+\beta_{2,1}+\right.$ $\left.\beta_{1,3}+\beta_{2,2}\right)+\operatorname{tr}(f)$ to (27), we get

$$
\left(m_{1}-1\right)\left(\beta_{1,1}+\beta_{2,1}\right)-\left(m_{1}+2\right)\left(\beta_{1,3}+\beta_{2,2}\right)=-\operatorname{tr}(f) .
$$

By denoting a cubic subfield fixed by $\eta$ by $M_{3}$, it means

$$
\left(m_{1}-1\right) \operatorname{tr}_{K / M_{3}}\left(\beta_{1,1}\right)-\left(m_{1}+2\right) \operatorname{tr}_{K / M_{3}}\left(\beta_{1,3}\right)=-\operatorname{tr}(f),
$$

which completes the proof, putting

$$
c=-\left(m_{1}+2\right) /\left(m_{1}-1\right), \quad \alpha=\beta_{1,1}, \quad \alpha^{\prime}=\beta_{1,3} .
$$

In [3], there are examples of a polynomial of type 2,3 , but at that time the author did not recognize any $S_{3}$-type polynomial satisfying the last condition in Proposition 5. In the next section, we give examples.

Let us give a basis of linear relations of an irreducible abelian polynomial of degree 6 without proof to describe a set $\mathfrak{D}(f, \sigma)$.

Proposition 6. Let $f$ be an irreducible polynomial of degree 6 with a root $\alpha_{1}$ and suppose that $\mathbb{Q}\left(\alpha_{1}\right) / \mathbb{Q}$ is an abelian extension of $\mathbb{Q}$ and let $\sigma$ be an automorphism satisfying $\sigma\left(\alpha_{i}\right)=\alpha_{i+1}\left(\alpha_{i}=\alpha_{j}\right.$ for $\left.i \equiv j \bmod 6\right)$ for roots $\alpha_{i}$ of $f$. Then a basis of linear relations among roots are:

(a) In case that $f$ is indecomposable and neither of type 2 nor of type 3.

$$
\sum_{i=1}^{6} \alpha_{i}=\operatorname{tr}(f) .
$$

(b) in case that $f$ is indecomposable and of type 2 ,

$$
\alpha_{1}+\alpha_{3}+\alpha_{5}=\alpha_{2}+\alpha_{4}+\alpha_{6}=\operatorname{tr}(f) / 2 .
$$

(c) In case that $f$ is indecomposable and of type 3 ,

$$
\sum \alpha_{i}=\operatorname{tr}(f), \alpha_{1}+\alpha_{2}-\left(\alpha_{4}+\alpha_{5}\right)=0, \alpha_{2}+\alpha_{3}-\left(\alpha_{5}+\alpha_{6}\right)=0 .
$$

(d) In case that $f(x)=g(h(x))$ is possible for a cubic polynomial $g$, but impossible for a quadratic polynomial $\mathrm{g}$,

$$
\alpha_{1}+\alpha_{4}=\alpha_{2}+\alpha_{5}=\alpha_{3}+\alpha_{6}=\operatorname{tr}(f) / 3 .
$$


(e) In case that $f(x)=g(h(x))$ is possible for a quadratic polynomial $g$, but impossible for a cubic polynomial $\mathrm{g}$,

$$
\alpha_{1}+\alpha_{3}+\alpha_{5}=\alpha_{2}+\alpha_{4}+\alpha_{6}=\operatorname{tr}(f) / 2 .
$$

(f) In case that $f$ is decomposable and $f(x)=g(h(x))$ is possible for both $\operatorname{deg} g=2,3$,

$$
\alpha_{1}+\alpha_{4}=\alpha_{2}+\alpha_{5}=\alpha_{3}+\alpha_{6}=\operatorname{tr}(f) / 3, \alpha_{1}-\alpha_{2}+\alpha_{3}=\operatorname{tr}(f) / 6 .
$$

Except this abelian case, even the classification of non-trivial relations is incomplete.

\section{Expectation 2 for a polynomial of degree 6}

We have no data to deny Expectation 2 for an irreducible and indecomposable polynomial $f$ in the case of degree $\leq 5$, but it fails in the case of degree 6 . The two conditions irreducibility and indecomposability are equivalent to having no non-trivial linear relations among roots in the case of degree $\leq 5$ as in the previous section. Data in [2] are less accurate in the case of degree 6 , that is $X$ in (11) was too small to guess the precise limit. We improve a method to guess the limit from approximate values.

Suppose that the limit in (11) exists and every $\operatorname{Pr}\left(f, L,\left\{R_{i}\right\}\right)$ is rational: Then for the common denominator $b$, we see that

$$
\sum_{\left\{R_{i}\right\}} \operatorname{Pr}\left(f, L,\left\{R_{i}\right\}\right) \cdot b=b, \operatorname{gcd}\left(\underset{\left\{R_{i}\right\}}{\operatorname{gcd}}\left(\operatorname{Pr}\left(f, L,\left\{R_{i}\right\}\right) \cdot b\right), b\right)=1 .
$$

Supposing that $b$ is less than 30000 and taking the above into account, let us consider integers $d$ with $1 \leq d \leq 30000$, which satisfies

$\sum_{\left\{R_{i}\right\}} r\left(\operatorname{Pr}_{X}\left(f, L,\left\{R_{i}\right\}\right) \cdot d\right)=d, \operatorname{gcd}\left(\underset{\left\{R_{i}\right\}}{\operatorname{gcd}}\left(r\left(\operatorname{Pr}_{X}\left(f, L,\left\{R_{i}\right\}\right) \cdot d\right)\right), d\right)=1$,

where $r(x)$ is an integer closest to $x$. Because, they must be satisfied if $d$ is the common denominator of $\operatorname{Pr}\left(f, L,\left\{R_{i}\right\}\right)$ and an approximation by $\operatorname{Pr}_{X}\left(f, L,\left\{R_{i}\right\}\right)$ is sufficiently well. We consider the following four measures, abbreviating $\operatorname{Pr}_{X}\left(f,[3] L,\left\{R_{i}\right\}\right)$ to $\operatorname{Pr}_{X, R_{i}}$ :

$$
\begin{aligned}
& e r_{1}:=\max _{\left\{R_{i}\right\}}\left|\operatorname{Pr}_{X, R_{i}} \cdot d-r\left(\operatorname{Pr}_{X, R_{i}} \cdot d\right)\right|, \\
& e r_{2}:=\sum_{\left\{R_{i}\right\}}\left|\operatorname{Pr}_{X, R_{i}} \cdot d-r\left(\operatorname{Pr}_{X, R_{i}} \cdot d\right)\right|^{2},
\end{aligned}
$$




\section{YOSHIYUKI KITAOKA}

$$
\begin{aligned}
& e r_{3}:=\max _{\left\{R_{i}\right\}}\left|\operatorname{Pr}_{X, R_{i}}-r\left(\operatorname{Pr}_{X, R_{i}} \cdot d\right) / d\right|, \\
& e r_{4}:=\sum_{\left\{R_{i}\right\}}\left|\operatorname{Pr}_{X, R_{i}}-r\left(\operatorname{Pr}_{X, R_{i}} \cdot d\right) / d\right|^{2} .
\end{aligned}
$$

If $\operatorname{Pr}_{X}\left(f, L,\left\{R_{i}\right\}\right)$ approximates a rational number $a / b$ well, they are close to 0 for $d=b$. So, we can find the conjectural denominator $b$ of $\operatorname{Pr}\left(f, L,\left\{R_{i}\right\}\right)$ by checking that there is a large number $X$ satisfying that there is an integer $d$ with $1 \leq d \leq 30000$ which gives the common minimum for four measures above. The first two $d$-adic measures $e r_{1}, e r_{2}$ seem to be more appropriate.

We put

$$
\begin{aligned}
& f_{1}:=x^{6}+2 x^{5}+4 x^{4}+x^{3}+2 x^{2}-3 x+1, \\
& f_{2}:=x^{6}+4 x^{5}+16 x^{4}+22 x^{3}+39 x^{2}+16 x+29 \\
& f_{3}:=x^{6}+5 x^{5}+11 x^{4}+13 x^{3}+23 x^{2}+31 x+43 \\
& f_{4}:=x^{6}+8 x^{5}+43 x^{4}+134 x^{3}+372 x^{2}+596 x+953 .
\end{aligned}
$$

They are irreducible and indecomposable and define the same cyclotomic field $\mathbb{Q}\left(\zeta_{7}\right)$, and the type of $f_{1}, f_{2}$ is 2 and that of $f_{3}, f_{4}$ is 3 .

Let $L=2$. A 6 -tuple $\left(R_{1}, \ldots, R_{6}\right)$ with $0 \leq R_{i} \leq L-1$ corresponds to an integer $r$ with $1 \leq r \leq L^{6}$ by

$$
r=1+\sum_{i=1}^{6} R_{i} L^{i-1}
$$

(I) The case that there is no non-trivial linear relation among roots :

For a polynomial $f=x^{6}+5 x^{5}+1$ in [2, data were insufficient. Wrong values $7 / 512=0.0136 \ldots, 9 / 512=0.0175 \ldots$ on p.87 in [2] are close to the conjectural values $13 / 960=0.0135 \ldots, 17 / 960=0.0177 \ldots$ on p.84 respectively. The common denominator for four measures above is 960 for $X=1.36 \cdot 10^{12}$ and the density matches with the conjecture (12). The density in (12) for $L=2$ is given explicitly by

$$
\operatorname{Pr}\left(f, 2,\left\{R_{i}\right\}\right)=\left\{\begin{array}{lll}
13 / 960 & \text { if } & \operatorname{tr}(f)+\sum R_{i} \equiv 0 \bmod 2 \\
17 / 960 & \text { if } \quad \operatorname{tr}(f)+\sum R_{i} \equiv 1 \bmod 2
\end{array}\right.
$$

(II) Case that $f$ is irreducible and indecomposable, and the type number is 2 . For $f=f_{1}, f_{2}$, the common denominator of $\operatorname{Pr}_{X}\left(f, 2,\left\{R_{i}\right\}\right)$ for four measures is 2304 , which is attained for $X=2 \cdot 10^{11}$. The following table of densities is arranged in the order of $r$ above. For example, $\left(R_{1}, \ldots, R_{6}\right)=(0, \ldots, 0)$ corresponds to $r=1$, and hence the density $\operatorname{Pr}\left(f_{1}, 2,(0, \ldots, 0)\right)$ is the first entry $36 / 2304$. 
$\operatorname{Pr}\left(f_{1}, 2,\left\{R_{i}\right\}\right)=[36,4,15,43,43,42,23,62,29,30,35,48,36,38,49,43$, $57,29,36,38,37,40,29,42,49,54,43,30,23,29,36,4$, $68,36,43,49,42,29,18,23,30,43,32,35,34,36,43,15$, $29,23,34,36,24,37,42,43,10,49,30,29,29,57,68,36] / 2304$,

$\begin{aligned} \operatorname{Pr}\left(f_{2}, 2,\left\{R_{i}\right\}\right)= & {[36,68,57,29,29,30,49,10,43,42,37,24,36,34,23,29,} \\ & 15,43,36,34,35,32,43,30,23,18,29,42,49,43,36,68, \\ & 4,36,29,23,30,43,54,49,42,29,40,37,38,36,29,57, \\ & 43,49,38,36,48,35,30,29,62,23,42,43,43,15,4,36] / 2304 .\end{aligned}$

Errors are

$$
e r_{1}\left(f_{1}\right)=0.019615 \operatorname{er}_{1}\left(f_{2}\right)=0.026945 \text {. }
$$

The density of another polynomial $f$ of type 2 for $L=2$ seems to be given by the above according to $\operatorname{tr}(f) \bmod 4 .(\operatorname{tr}(f)$ is an even integer in this case.) Let $f(x)$ be of type 2 ; then a polynomial $f(x-1)$ is also of type 2 and

$$
\operatorname{tr}(f(x-1)) \equiv \operatorname{tr}(f(x))+2 \bmod 4 \quad \text { is easy. }
$$

If $r_{1}, \ldots, r_{6}$ are local roots with $r_{i} \equiv R_{i} \bmod 2$, then $r_{1}^{\prime}:=r_{1}+1, \ldots, r_{6}^{\prime}:=r_{6}+1$ are also local roots of $f(x-1)$ with $r_{i}^{\prime} \equiv R_{i}+1 \bmod 2$. It is the reason why the densities $\operatorname{Pr}\left(f_{1}, 2,\left\{R_{i}\right\}\right), \operatorname{Pr}\left(f_{2}, 2,\left\{R_{i}\right\}\right)$ are anti-symmetric. Some properties of $f=f_{1}, f_{2}$ are for $R=\left(R_{1}, \ldots, R_{6}\right), R^{\prime}=\left(R_{1}^{\prime}, \ldots, R_{6}^{\prime}\right)$,

(1) if $R_{i}+R_{i}^{\prime}=1$ for $1 \leq{ }^{\forall} i \leq 6$, then $\operatorname{Pr}\left(f, 2,\left\{R_{i}\right\}\right)+\operatorname{Pr}\left(f, 2,\left\{R_{i}^{\prime}\right\}\right)=$ $72 / 2304=1 / 32$,

(2) $\sum_{\sum R_{i} \equiv 0 \bmod 2} \operatorname{Pr}\left(f, 2,\left\{R_{i}\right\}\right)=\sum_{\sum R_{i} \equiv 1 \bmod 2} \operatorname{Pr}\left(f, 2,\left\{R_{i}\right\}\right)=1 / 2$,

(3) if $R_{i}=1-R_{7-i}^{\prime}$ for $1 \leq{ }^{\forall} i \leq 6$, then $\operatorname{Pr}\left(f, 2,\left\{R_{i}\right\}\right)=\operatorname{Pr}\left(f, 2,\left\{R_{i}^{\prime}\right\}\right)$.

The third property is explained as follows: If we have $f(x) \equiv \prod\left(x-r_{i}\right) \bmod p$, then $f(-x) \equiv \prod\left(x-r_{i}^{\prime}\right) \bmod p$ with $r_{i}^{\prime}=p-r_{7-i}$ is easy to see. Hence the condition $r_{i} \equiv R_{i} \bmod 2$ implies $r_{i}^{\prime} \equiv 1-R_{7-i} \bmod 2$ for an odd prime $p$.

The author does not know how to give densities directly from $\left\{R_{i}\right\}$.

A basis of linear relations of $f=f_{1}, f_{2}$ with an appropriate numbering is

$$
\alpha_{1}+\alpha_{3}+\alpha_{5}=\alpha_{2}+\alpha_{4}+\alpha_{6}=\operatorname{tr}(f) / 2 .
$$

(III) The case that $f$ is irreducible and indecomposable, and the type number is 3 . For $f=f_{3}, f_{4}$, the common denominator of $\operatorname{Pr}_{X}\left(f, 2,\left\{R_{i}\right\}\right)$ for four measures is 15120 , which is attained for $X=2 \cdot 10^{11}$. The following table of densities is 


\section{YOSHIYUKI KITAOKA}

arranged in the order of $r$ as above.

$$
\begin{aligned}
\operatorname{Pr}\left(f_{3}, 2,\left\{R_{i}\right\}\right)= & {[525,189,63,414,63,229,176,159,63,224,288,172,} \\
& 544,125,204,414,63,153,394,125,288,320,300,229, \\
& 176,401,300,224,204,153,189,189,189,189,153,204, \\
& 224,300,401,176,229,300,320,288,125,394,153,63, \\
& 414,204,125,544,172,288,224,63,159,176,229,63, \\
& 414,63,189,525] / 15120, \\
\operatorname{Pr}\left(f_{4}, 2,\left\{R_{i}\right\}\right)=[ & 420,288,180,279,162,157,140,336,162,110,174,229, \\
& 343,176,273,279,180,84,247,176,174,485,405,157, \\
& 140,602,405,110,273,84,42,288,288,42,84,273, \\
& 110,405,602,140,157,405,485,174,176,247,84,180, \\
& 279,273,176,343,229,174,110,162,336,140,157,162, \\
& 279,180,288,420] / 15120 .
\end{aligned}
$$

Errors are

$$
\operatorname{er}_{1}\left(f_{3}\right)=0.16450, \operatorname{er}_{1}\left(f_{4}\right)=0.16892 .
$$

We note that $\operatorname{tr}\left(f_{3}\right)$ is odd and $\operatorname{tr}\left(f_{4}\right)$ is even, and the density of another polynomial of type 3 for $L=2$ seems to be given by the above according to $\operatorname{tr}(f) \bmod 2$.

A basis of linear relations of $f=f_{3}, f_{4}$ is

$$
\sum \alpha_{i}=\operatorname{tr}(f), \alpha_{1}+\alpha_{2}=\alpha_{4}+\alpha_{5}, \alpha_{2}+\alpha_{3}=\alpha_{5}+\alpha_{6}
$$

(IV) The case that $K=\mathbb{Q}\left(\alpha_{1}\right)$ is an $S_{3}$-extension and there are a rational number $c$, two distinct roots $\alpha, \alpha^{\prime}$ of $f$, and a cubic subfield $M_{3}$ such that $\operatorname{tr}_{K / M_{3}}(\alpha)+c \cdot \operatorname{tr}_{K / M_{3}}\left(\alpha^{\prime}\right) \in \mathbb{Q}$. Let us give two examples:

The first example is $f=x^{6}+3$. Putting $y:=\sqrt[6]{-3}$ with $y^{3}=\sqrt{-3}$, roots are

$$
\begin{aligned}
& \beta_{1,1}=y, \quad \beta_{1,2}=(-1+\sqrt{-3}) / 2 \cdot y, \quad \beta_{1,3}=-(1+\sqrt{-3}) / 2 \cdot y, \\
& \beta_{2,1}=-y, \quad \beta_{2,2}=(1-\sqrt{-3}) / 2 \cdot y, \quad \beta_{2,3}=(1+\sqrt{-3}) / 2 \cdot y .
\end{aligned}
$$

Automorphisms $\eta, \sigma$ in the proof of Proposition [5] are given by $y \mapsto-y, y \mapsto$ $(-1+\sqrt{-3}) / 2 \cdot y$, respectively. A basis of linear relations is four equations

$$
\beta_{1,2}=-\beta_{1,1}+\beta_{2,3}, \quad \beta_{1,3}=-\beta_{2,3}, \quad \beta_{2,1}=-\beta_{1,1}, \quad \beta_{2,2}=\beta_{1,1}-\beta_{2,3} .
$$

The inclusion $\operatorname{tr}_{K / M_{3}}(\alpha)+c \cdot \operatorname{tr}_{K / M_{3}}\left(\alpha^{\prime}\right) \in \mathbb{Q}$ is obvious for $\alpha=y, c=0$. Densities $\operatorname{Pr}\left(f, 2,\left\{R_{i}\right\}\right)$ for $\left[R_{1}, \ldots, R_{6}\right]$ are given by:

$$
\left\{\begin{array}{ccl}
1 / 16 & \text { for } & {[1,1,1,0,0,0],[1,0,0,1,1,0],[0,1,0,1,0,1],[0,0,1,0,1,1]} \\
3 / 16 & \text { for } & {[1,1,0,1,0,0],[1,0,1,0,1,0],[0,1,1,0,0,1],[0,0,0,1,1,1]} \\
0 & & \text { otherwise. }
\end{array}\right.
$$




\section{DISTRIBUTION OF ROOTS MODULO A PRIME}

Since $-r$ is also a root for a local root $r$, a relation $r_{i}=p-r_{7-i}$ should hold for $i=1,2,3$, hence $R_{i}+R_{7-i} \equiv 1 \bmod 2$, i.e., $R_{i}+R_{7-i}=1$. This elucidates the cases of density 0 .

The second example is $f=x^{6}+100 x^{4}-168 x^{3}+5200 x^{2}+16800 x+26256$, and let $\beta$ be a root. Then we see that roots $\alpha_{1}, \ldots, \alpha_{6}$ of $f$ are in order $-1 / 2122960$ times $-2122960 \beta$,

$$
\begin{array}{rrrrrrr}
-1757 \beta^{5} & +2758 \beta^{4} & -189756 \beta^{3} & +699188 \beta^{2} & -11117792 \beta & -9582496 \\
-1463 \beta^{5} & +182 \beta^{4} & -158004 \beta^{3} & +159292 \beta^{2} & -8902208 \beta & -22357664 \\
125 \beta^{5} & +710 \beta^{4} & +13500 \beta^{3} & +131500 \beta^{2} & +2458400 \beta & +6844000 \\
1088 \beta^{5} & -2312 \beta^{4} & +117504 \beta^{3} & -553792 \beta^{2} & +7895888 \beta & +1825664 \\
2007 \beta^{5} & -1338 \beta^{4} & +216756 \beta^{3} & -436188 \beta^{2} & +11788672 \beta & +23270496 .
\end{array}
$$

The polynomial $f$ is irreducible, indecomposable and not of type 2,3 , and we see

$$
\alpha_{1}+\alpha_{6}+3\left(\alpha_{2}+\alpha_{5}\right)=0
$$

and

$$
\left(\alpha_{1}+\alpha_{6}\right)^{3}=756=28 \cdot 3^{3}
$$

and

$$
\left(\alpha_{1}+\alpha_{5}\right)^{3}=-224=-28 \cdot 2^{3} .
$$

Hence $\alpha_{1}+\alpha_{6}$ is a trace to a cubic subfield defined by $x^{3}-756=0$, hence

$$
\operatorname{tr}_{K / M_{3}}(\alpha)+c \cdot \operatorname{tr}_{K / M_{3}}\left(\alpha^{\prime}\right) \in \mathbb{Q} \quad \text { for } \quad \alpha=\alpha_{1}, \quad c=3, \quad \alpha^{\prime}=\alpha_{2} .
$$

A basis of linear relations is three equations

$$
\sum_{i} \alpha_{i}=0, \alpha_{1}+\alpha_{6}+3\left(\alpha_{2}+\alpha_{5}\right)=0, \alpha_{1}+\alpha_{5}-2\left(\alpha_{3}+\alpha_{6}\right)=0 .
$$

And we see $\left(\alpha_{3}+\alpha_{6}\right)^{3}=-28$. The speed of convergence is slow,

$$
e r_{1}(f)>0.3 \text { even for } X=4 \cdot 10^{13} \text {. }
$$

The author checked the following: Let us consider following 16 polynomials

$$
\begin{array}{ll}
x^{6}-9 x^{4}-4 x^{3}+9 x^{2}+3 x-1, & x^{6}-2 x^{3}+9 x^{2}+6 x+2, \\
x^{6}-7 x^{3}-6 x^{2}-9 x-3, & x^{6}-10 x^{4}-4 x^{3}+10 x^{2}-1, \\
x^{6}-9 x^{4}-8 x^{3}+6 x^{2}+6 x+1, & x^{6}-10 x^{4}-7 x^{3}+10 x^{2}-1, \\
x^{6}-7 x^{4}+8 x^{2}-10 x+1, & x^{6}-x^{4}-2 x^{3}+7 x^{2}+x+10, \\
x^{6}-8 x^{4}-10 x^{3}-3 x^{2}+2 x+6, & x^{6}-5 x^{4}-7 x^{3}-3 x^{2}-x+3, \\
x^{6}-10 x^{4}-10 x^{3}-10 x^{2}+1, & x^{6}-9 x^{4}-10 x^{3}-2 x^{2}-1,
\end{array}
$$




\section{YOSHIYUKI KITAOKA}

$$
\begin{array}{ll}
x^{6}-10 x^{4}-10 x^{3}-10 x^{2}-7, & x^{6}-10 x^{4}-8 x^{3}-4 x^{2}+8 x-2, \\
x^{6}-10 x^{4}-10 x^{3}+5 x^{2}-2 x+9, & x^{6}-10 x^{4}-10 x^{3}-10 x^{2}-10 x-10
\end{array}
$$

which exhaust all types of Galois closure, checked by pari/gp. Take a root $\alpha$ of one of them and a polynomial $f$ whose root is

$$
\sum_{i=1}^{6} c_{i} \alpha^{i-1} \quad \text { with } \quad-1 \leq c_{i} \leq 1 .
$$

We consider irreducible and indecomposable ones of degree 6 only. We checked densities $\operatorname{Pr}_{X}\left(f, 2,\left\{R_{i}\right\}\right)$ approximate well densities of special polynomials $f_{1}, f_{2}, f_{3}, f_{4}$ or (29), where we say that for a rational number $a / b$ and a real number $x, x$ approximates well $a / b$ if the nearest integer $r(b x)$ to $b x$ is $a$, i.e., $r(b x)=a$.

For a polynomial $f=x^{6}-3 x^{5}+6 x^{4}+3 x^{3}-9 x^{2}-18 x+36$ of type 3 and $\operatorname{tr}(f) \equiv 1 \bmod 2$, which is given in [2, (5) on p.87], $\operatorname{Pr}_{X}\left(f, 2,\left\{R_{i}\right\}\right)\left(X \leq 10^{11}\right)$ approximates well densities given above and the densities on p.87 in [2] should be corrected by the above.

\section{REFERENCES}

[1] DUKE, W.-FRIEDLANDER, J. B.-IWANIEC, H.: Equidistribution of roots of a quadratic congruence to prime moduli, Ann. of Math. (2) 141 (1995), no. 2, 423-441.

[2] HADANO, T.-KITAOKA, Y.-KUBOTA, T.-NOZAKI, M.: Densities of sets of primes related to decimal expansion of rational numbers. (W. Zhang and Y. Tanigawa, eds.) In: Number Theory: Tradition and Modernization, The 3rd China-Japan seminar on number theory, Xi'an, China, February 12-16, 2004. Developments. Math. Vol. 15, 2006, Springer, New York, pp. 67-80,

[3] KITAOKA, Y.: A statistical relation of roots of a polynomial in different local fields, Math. Comput. 78 (2009), no. 265, 523-536.

[4] A statistical relation of roots of a polynomial in different local fields II, (Aoki, Takashi ed. et al.) In: Number Theory: Dreaming in Dreams, Proceedings of The 5th China-Japan seminar, Higashi-Osaka, Japan, August 27-31, 2008. Ser. Number Theory Appl. Vol. 6, 2010, World Sci. Publ., Hackensack, NJ, pp. 106-126.

[5] A statistical relation of roots of a polynomial in different local fields III, Osaka J. Math. 49 (2012), 393-420.

[6] Statistical distribution of roots of a polynomial modulo prime powers, In: Number Theory: Plowing and Starring through High Wave Forms, Ser. Number Theory Appl. Vol. 11, 2015, World Sci. publ., Hackensack, NJ, pp. 75-94.

[7] _ Statistical distribution of roots of a polynomial modulo primes, (submitted). 


\section{DISTRIBUTION OF ROOTS MODULO A PRIME}

[8] Y. KITAOKA: Statistical distribution of roots of a polynomial modulo primes II, Unif. Distrib. Theory 12 (2017), no. 1, 109-122.

[9] ТÓTH, T. Á.: Roots of Quadratic congruences, Internat. Math. Res. Notices 2000, no. 14, (2000) 719-739.

Received October 5, 2016

Accepted January 19, 2017

\section{Yoshiyuki Kitaoka}

Uzunawa 1085-10,

Asahi-cho,

Mie, 510-8104

JAPAN

E-mail: kitaoka@meijo-u.ac.jp 